\title{
DYNAMICAL COLLAPSE OF NONROTATING MAGNETIC MOLECULAR CLOUD CORES: EVOLUTION THROUGH POINT-MASS FORMATION
}

\author{
Glenn E. Ciolek and Arieh Königl \\ Department of Astronomy and Astrophysics, University of Chicago \\ 5640 S. Ellis Avenue, Chicago, IL 60637 \\ Accepted for publication in The Astrophysical Journal, 1 Sept 1998 issue
}

\begin{abstract}
We present a numerical simulation of the dynamical collapse of a nonrotating, magnetic molecular cloud core and follow the core's evolution through the formation of a central point mass and its subsequent growth to a $1 M_{\odot}$ protostar. The epoch of point-mass formation (PMF) is investigated by a self-consistent extension of previously presented models of core formation and contraction in axisymmetric, self-gravitating, isothermal, magnetically supported interstellar molecular clouds. Prior to PMF, the core is dynamically contracting and is not well approximated by a quasistatic equilibrium model. Ambipolar diffusion, which plays a key role in the early evolution of the core, is unimportant during the dynamical pre-PMF collapse phase. However, the appearance of a central mass, through its effect on the gravitational field in the inner core regions, leads to a "revitalization" of ambipolar diffusion in the weakly ionized gas surrounding the central protostar. This process is so efficient that it leads to a decoupling of the field from the matter and results in an outward-propagating hydromagnetic C-type shock. The existence of an ambipolar diffusion-mediated shock of this type was predicted by Li \& McKee (1996), and we find that the basic shock structure given by their analytic model is well reproduced by our more accurate numerical results. Our calculation also demonstrates that ambipolar diffusion, rather than Ohmic diffusivity operating in the innermost core region, is the main field decoupling mechanism responsible for driving the shock after PMF.

The passage of the shock leads to a substantial redistribution, by ambipolar diffusion but possibly also by magnetic interchange, of the mass contained within the magnetic flux tubes in the inner core. In particular, ambipolar diffusion reduces the flux initially threading a collapsing $\sim 1 M_{\odot}$ core by a factor $\gtrsim 10^{3}$ by the time this mass accumulates within the inner radius $(\simeq 7.3 \mathrm{AU})$ of our computational grid. This reduction, which occurs primarily during
\end{abstract}


the post-PMF phase of the collapse, represents a significant step towards the resolution of the protostellar magnetic flux problem.

Our calculations indicate that a $1 M_{\odot}$ protostar forms in $\sim 1.5 \times 10^{5} \mathrm{yr}$ for typical cloud parameters. The mass accretion rate is time dependent, in part because of the $\mathrm{C}$-shock that decelerates the infalling matter as it propagates outward: the accretion rate rises to $\simeq 9.4 M_{\odot} \mathrm{Myr}^{-1}$ early on and decreases to $\simeq 5.6 M_{\odot} \mathrm{Myr}^{-1}$ by the time a solar-mass protostar is formed. The infalling gas disk surrounding the protostar has a mass $\sim 10^{-2} M_{\odot}$ at radii $r \gtrsim 500 \mathrm{AU}$. A distinguishing prediction of our model is that the rapid ambipolar diffusion after the formation of a protostar should give rise to large $\left(\gtrsim 1 \mathrm{~km} \mathrm{~s}^{-1}\right)$, and potentially measurable, ion-neutral drift speeds on scales $r \lesssim 200$ AU.

The main features of our simulation, including the C-shock formation after PMF, are captured by a similarity solution that incorporates the effects of ambipolar diffusion (Contopoulos, Ciolek, \& Königl 1997).

Subject headings: accretion, accretion disks — diffusion - ISM:

clouds - ISM: magnetic fields — MHD — stars: formation — stars:

pre-main-sequence

\section{Introduction}

It is generally accepted that most of the star-formation activity in our galaxy takes place through the gravitational collapse of molecular cloud cores (e.g., Mouschovias 1987; Shu, Adams, \& Lizano 1987). It is, furthermore, believed that interstellar magnetic fields play a central role in this process in that their stresses are the dominant agent that acts against gravity to prevent, or delay, cloud collapse (e.g., Mouschovias 1978; McKee et al. 1993). This is embodied in the concept of a critical mass $M_{\text {crit }}$, which in general takes account of both the magnetic and the thermal pressure contributions to the support of the cloud, but which, in the case that magnetic stresses dominate, reduces to $M_{\text {crit }} \approx 0.13 \Phi_{B} / G^{1 / 2}$, where $G$ is the gravitational constant and $\Phi_{B}$ is the magnetic flux that threads the cloud. Clouds whose mass $M$ exceeds $M_{\text {crit }}$ are "supercritical": they collapse on the free-fall timescale. In contrast, "subcritical" clouds $\left(M<M_{\text {crit }}\right)$ can avoid collapse on the much longer ambipolar diffusion timescale. In the latter case, the neutrals gradually contract by diffusing inward through the ions and field, leaving behind a magnetically supported envelope and eventually forming a supercritical core that undergoes dynamical collapse (e.g., Mouschovias 1996).

Because of the complexity of the problem — it involves solving the full dynamical equations of a magnetized, multicomponent (neutrals, ions, electrons, as well as charged and neutral grains) fluid that evolves over many decades in size and density in a nonspherically 
symmetric manner (because of the presence of ordered magnetic fields and likely also rotation) - much of the progress in this area has been accomplished through the use of numerical simulations. One of the main efforts to simulate core formation and contraction due to ambipolar diffusion in magnetically supported molecular clouds has been carried out by Mouschovias and coworkers (e.g., Fiedler \& Mouschovias 1992, 1993; Ciolek \& Mouschovias 1993, 1994, 1995, hereafter CM93, CM94, CM95, respectively; Basu \& Mouschovias 1994, 1995a, b). These studies followed the evolution of the core over six decades in density up to central densities $\sim 3 \times 10^{9} \mathrm{~cm}^{-3}$, where the assumption of isothermality starts to break down because of radiative trapping (e.g., Gaustad 1963; Hayashi 1966). This assumption had been adopted in the interest of simplicity: sophisticated and computationally intensive numerical techniques are generally needed to calculate the thermal structure of the gas during the opaque phase of protostellar evolution (e.g., Larson 1969, 1972; Winkler \& Newman 1980; Stahler, Shu, \& Taam 1981; Boss 1984; Myhill \& Kaula 1992; Myhill \& Boss 1993). As a result of this restriction, the aforementioned calculations did not follow the collapse of the core to the time where a point mass - a protostar - is formed at the center, although they did obtain valuable information on the conditions leading to this critical event. In particular, by the time these simulations were terminated, the inner region of the core was collapsing dynamically and was characterized by neutral infall speeds $\sim C$ (the isothermal speed of sound) and inward accelerations $\gtrsim 0.3\left|g_{r}\right|$ [where $g_{r}(r)$ is the local gravitational acceleration]. Furthermore, the thermal pressure, while remaining relatively unimportant in the envelope, came to exceed the magnetic pressure near the center. Basu (1997) derived a time-dependent, semianalytic solution that extended these ambipolar diffusion models up to the instant of point-mass formation (hereafter referred to as PMF D). He found that ambipolar diffusion continues to gradually erode the retarding magnetic forces in the inner core, making the collapse increasingly more dynamical (and the thermal-to-magnetic pressure ratio in the inner core progressively larger) as PMF is approached.

The diminution of magnetic forces in the innermost regions of a collapsing core just prior to PMF suggests that one could gain some insight into the protostar formation process from previous studies of PMF in nonmagnetic, spherically symmetric, isothermal clouds. Analytic similarity solutions have uncovered two limiting behaviors: Penston (1969) and Larson (1969) found a solution in which, just before PMF, the infall speed approaches $\sim 3.3 C$ at all radii while the density scales with radius as $r^{-2}$, resulting in a spatially

\footnotetext{
${ }^{1}$ Creation of a central point mass was commonly referred to in previous papers as "core formation." In this paper we use the term "point-mass formation" so as not to confuse this process with the formation of an extended, magnetically supercritical, molecular cloud core.
} 
uniform mass inflow rate $\sim 29 C^{3} / G$ (where $G$ is the gravitational constant). Hunter (1977) extended this solution past PMF and showed that, immediately after the central mass is formed, the accretion rate onto the protostar increases to $\sim 47 C^{3} / G$. In the other limit, Shu (1977) obtained a solution that is static prior to PMF (with the density distribution of a singular isothermal sphere, which also scales as $r^{-2}$ ) and that takes on an expansion-wave character (with a constant mass accretion rate $\sim 0.98 C^{3} / G$ onto the protostar) following PMF. Hunter (1977) and Whitworth \& Summers (1985) demonstrated that there are, in fact, infinitely many similarity solutions that span the range between the Larson-Penston and Shu results, with the nature of any given solution being determined by the initial configuration of the cloud and the conditions at its boundary. Numerical simulations carried out by Hunter (1977) and by Foster \& Chevalier (1993) confirmed the dependence on the initial and boundary conditions. In particular, it was found that the behavior of the central regions of clouds that are initially marginally stable to collapse approximates that of the Larson-Penston solution at the PMF epoch, although it was determined that the mass accretion rate onto the protostar declines at later times. It was, however, also found that the post-PMF evolution of clouds that initially have more extended envelopes approximates that of the Shu solution at late times. Since the initial and boundary conditions of real clouds are expected to depend on the detailed configuration and evolution of the embedded magnetic field, it is clear that one needs to incorporate magnetic field effects into the collapse calculations to adequately model the formation of protostars.

There have been several recent attempts to calculate PMF following the collapse of magnetic interstellar clouds. Although they have all contributed to our understanding of the processes involved, their results were hampered by the adopted assumptions or approximations. For example, Tomisaka (1996) modeled clouds that had equal thermal and magnetic energy densities, so that they were not primarily supported by magnetic fields. This means that his model clouds were magnetically supercritical. This assumption is at variance with $\mathrm{H} \mathrm{I}$ and $\mathrm{OH}$ Zeeman measurements of magnetic field strengths in molecular clouds, which are consistent with models of magnetically subcritical clouds (Crutcher et al. 1993, 1994, 1996). Li \& Shu (1997) modeled PMF in self-similar, magnetic cores. They assumed that cores immediately before PMF can be represented by hydrostatic configurations of singular isothermal disks and that the magnetic flux is frozen into the neutrals. These assumptions are inconsistent with the above-cited results of numerical simulations and semianalytic solutions of the collapse of magnetically supported molecular clouds that undergo ambipolar diffusion (as well as with the simulations of thermally supported spherical clouds), which have found that the inner core regions collapse dynamically as PMF is approached (see also §3.2). As we show below, ambipolar diffusion, which plays a key role in bringing about the dynamical collapse, is generally important also 
after PMF. Safier, McKee, \& Stahler (1997) did include ambipolar diffusion in the modeling of magnetic cloud collapse and post-PMF evolution. However, their model is strictly spherical, and they neglected the effect of thermal pressure gradients in comparison with magnetic stresses. Furthermore, their formulation is quasistatic and does not incorporate the magnetic induction equation for the time evolution of the magnetic field. Li (1998) extended the Safier et al. model by including thermal-pressure and time-dependent terms and by adding the induction equation. This enabled him to follow the time evolution of his model cores (for $r>150$ AU) even during the dynamical phases of the collapse. However, by retaining the spherical-symmetry assumption of Safier et al., his calculations were also unable to yield the geometry of the magnetic field lines. [f

The importance of ambipolar diffusion after PMF can be inferred by comparing the ambipolar diffusion timescale $\tau_{\mathrm{AD}}=r / v_{\mathrm{D}}$ (where $v_{\mathrm{D}}$ is the ion-neutral drift speed) and the gravitational contraction $\left(\simeq\right.$ free-fall) timescale $\tau_{\mathrm{gr}}=\left(r /\left|g_{r}\right|\right)^{1 / 2}$ before and after PMF. In axisymmetric geometry, $\tau_{\mathrm{AD}} / \tau_{\mathrm{gr}} \simeq\left(\tau_{\mathrm{gr}} / \tau_{\mathrm{ni}}\right) \mu_{B}{ }^{2}$ in the inner flux tubes of a supercritical core (e.g., Mouschovias 1991), where $\mu_{B}(r)$ is the total mass-to-flux ratio at radius $r$ (in units of the critical value for gravitational collapse) and

$$
\tau_{\mathrm{ni}}=1.4\left[1+0.067 \frac{\left(m_{\mathrm{H}_{2}} / 2 \text { a.m.u. }\right)}{\left(m_{\mathrm{i}} / 30 \text { a.m.u. }\right)}\right] \frac{1}{n_{\mathrm{i}}\langle\sigma w\rangle_{\mathrm{ni}}}
$$

is the neutral-ion collision time. In equation (11), $n_{\mathrm{i}}$ is the ion density and $\langle\sigma w\rangle_{\mathrm{ni}}$ is the average collisional rate between ions of mass $m_{\mathrm{i}}$ and neutral $\mathrm{H}_{2}$ molecules of mass $m_{\mathrm{H}_{2}}\left(\simeq 1.7 \times 10^{-9} \mathrm{~cm}^{3} \mathrm{~s}^{-1}\right.$ for collisions between neutrals and $\mathrm{Mg}^{+}$or $\mathrm{HCO}^{+}$ions; McDaniel \& Mason 1973); the factor 1.4 accounts for a $20 \%$ helium abundance by number. CM94 and CM95 found that, to first order for the late pre-PMF evolution of cores in disk-like clouds, the magnetic field $B \approx 3\left(r_{0} / r\right) \mathrm{mG}$ (where $\left.r_{0}=40 \mathrm{AU}\right), n_{\mathrm{i}} \approx 0.1 \mathrm{~cm}^{-3}$ (valid for neutral densities $n_{\mathrm{n}} \gtrsim 10^{7} \mathrm{~cm}^{-3}$; see Figs. $2 b$ and $4 b$ in CM94), the vertical column density $\sigma_{\mathrm{n}} \approx 5\left(r_{0} / r\right) \mathrm{g} \mathrm{cm}^{-2}$, and the total mass $M(r) \approx 6 \times 10^{-3}\left(r / r_{0}\right) M_{\odot}$. For a disk-like cloud, the critical mass-to-flux ratio $\left(M / \Phi_{B}\right)_{\mathrm{d} \text {,crit }}=\left(4 \pi^{2} G\right)^{-1 / 2}$, where $G$ is the gravitational constant (Nakano \& Nakamura 1978). Therefore, one finds $\mu_{B} \approx 2.7,\left|g_{r}\right| \approx G M(r) / r^{2} \approx 2 \times 10^{-6}\left(r_{0} / r\right) \mathrm{cm} \mathrm{s}^{-2}$, and $\tau_{\mathrm{ni}} \approx 7 \times 10^{9} \mathrm{~s}$, which yields $\tau_{\mathrm{gr}} \approx 2 \times 10^{10}\left(r / r_{0}\right)$ s and $\tau_{\mathrm{AD}} / \tau_{\mathrm{gr}} \approx 20\left(r / r_{0}\right)$. It follows that $\tau_{\mathrm{AD}} / \tau_{\mathrm{gr}} \gg 1$ for $r \gg 2$ AU. Hence, as has already been demonstrated in the past, ambipolar diffusion

\footnotetext{
${ }^{2}$ The same is true for the spherically symmetric self-similar model of a collapsing magnetic cloud devised by Chiueh \& Chou (1994), which, however, does not include ambipolar diffusion. It should be noted that all models that assume spherical symmetry do not satisfy the solenoidal condition $\nabla \cdot B=0$ on the magnetic field.
} 
is ineffective as a dynamically collapsing core approaches PMF, and for $r \gg 2$ AU the magnetic flux can be considered frozen into the neutrals. (For reasons discussed in $\S 2.2$, we do not consider $r \lesssim 5$ AU.) Turning now to the post-PMF epoch, when the central point mass comes to dominate the gravitational field in the innermost flux tubes, we use the relation $\tau_{\mathrm{AD}} / \tau_{\mathrm{gr}} \approx\left(1-\left|a_{\mathrm{n}}\right| /\left|g_{r}\right|\right)^{-1}\left(\tau_{\mathrm{gr}} / \tau_{\mathrm{ni}}\right)$, where $a_{\mathrm{n}}$ is the inward acceleration of the neutrals and $g_{r}$ is the total gravitational acceleration (see eqs. [14] and [15] in $\S 3.3)$. In this case, normalizing $r$ as before, $\left|g_{r}\right| \approx 4 \times 10^{-4}\left(M_{\text {cent }} / M_{\odot}\right)\left(r_{0} / r\right)^{2} \mathrm{~cm} \mathrm{~s}^{-2}$. Substituting again $\tau_{\mathrm{ni}} \approx 7 \times 10^{9} \mathrm{~s}$ (corresponding to $n_{\mathrm{i}} \approx 0.1 \mathrm{~cm}^{-3}$ ), the above ratio becomes $\tau_{\mathrm{AD}} / \tau_{\mathrm{gr}} \approx 0.2\left(r / r_{0}\right)^{3 / 2}\left(M_{\odot} / M_{\text {cent }}\right)^{1 / 2}\left(1-\left|a_{\mathrm{n}}\right| /\left|g_{r}\right|\right)^{-1}$. For $\left|a_{\mathrm{n}}\right| /\left|g_{r}\right|$ in the range 0.2 - 0.9, which corresponds to the period after PMF when the collapse becomes progressively more dynamical, one infers $\tau_{\mathrm{AD}} / \tau_{\mathrm{gr}} \approx(0.2-2)\left(r / r_{0}\right)^{3 / 2}\left(M_{\odot} / M_{\text {cent }}\right)^{1 / 2}$. Hence $\tau_{\mathrm{AD}} \lesssim \tau_{\mathrm{gr}}$ for $M_{\text {cent }} \gtrsim 0.1 M_{\odot}$ and $r \lesssim 50 \mathrm{AU}$. [A similar result is obtained if one continues to use the pre-PMF relations and simply substitutes $M_{\text {cent }}$ for $M(r)$.] This estimate indicates that decoupling of the gas and magnetic field by ambipolar diffusion should occur in the inner core regions after PMF. The physical reason for this is that the strength of the gravitational field in the weakly ionized gas near the origin is greatly enhanced by the appearance and growth of a central point mass, causing the neutrals to fall in more rapidly while the plasma and magnetic field are left behind. (The same basic reason - the appearance of a progressively growing free-fall zone around the origin following PMF — is also the cause of the increase in the mass inflow rate into the center at that epoch first discovered in the above-referenced nonmagnetic collapse calculations.) The foregoing conclusion is verified by a detailed calculation in $\S 3.3$, where we show that ambipolar diffusion after PMF is, in fact, so efficient that it effectively decouples the neutrals and magnetic field in the innermost core region, with dramatic consequences for the subsequent dynamical evolution of the core. An alternative, yet equivalent, analysis of the effectiveness of ambipolar diffusion following PMF, based on the scaling of magnetic forces (particularly the magnetic tension force) after $\mathrm{PMF}$, is given in Appendix $\mathrm{C}$.

In this paper we present a detailed numerical simulation of point-mass formation in a nonrotating, magnetic, dynamically collapsing protostellar core, properly accounting for the effect of ambipolar diffusion. Unlike earlier studies, we use an initial state that is consistent with the realistic models of core formation and collapse, as presented earlier by Mouschovias and coworkers. As we noted above, the simulations carried out by that group were terminated at densities where the isothermality assumption started to become invalid because of radiative trapping. Although a proper treatment of radiative trapping is indispensable for a complete treatment of the star-formation process, one can adopt a simpler approach that circumvents this difficulty by removing the region of radiative trapping from the active computation mesh. This is justified by the fact that the region 
of radiative trapping (typically a few $\mathrm{AU}$ ) is several orders of magnitude smaller than the characteristic core size $\left(r_{\text {core }} \approx 0.1 \mathrm{pc}\right)$. This region can therefore be considered to be effectively point-like, and one can proceed to calculate the formation of a central point mass within a gravitationally collapsing core and its effect on the subsequent evolution of the core by retaining the isothermality assumption. In adopting this approach, we note that the assumption of isothermality was also employed in previous studies of protostar formation in nonmagnetic cloud cores as well as in the more recent attempts to model PMF in magnetic clouds.

The plan of the paper is as follows. In $\S 2$ we review the main characteristics of the pre-PMF core-evolution calculations of CM93, CM94, and CM95 and outline the model modifications that we have implemented to extend the simulations beyond PMF. In $\S 3$ we present the results of our calculations. We consider a typical model and follow the evolution of all physical quantities of interest through the PMF epoch. We also describe the formation (due to ambipolar diffusion in the innermost core after PMF) of a C-type shock and consider its propagation through the collapsing core. The appearance of such a shock as a result of field-matter decoupling was first pointed out by Li \& McKee (1996), who proposed that the relevant field decoupling mechanism was Ohmic dissipation in the innermost regions of the core. Our study, however, reveals that ambipolar diffusion occurring outside the region of Ohmic dissipation is the main cause of magnetic flux decoupling after PMF. In $\S 4$ we discuss the structure of the ambipolar diffusion-mediated shock and show that our numerical calculations qualitatively reproduce the predictions of the simplified analytic model constructed by Li \& McKee (1996). We present a quantitative comparison with their results and also address the issue of interchange instability in the post-shock region, first raised in their work, in light of our detailed computations. In that section we also discuss the observational implications of our simulation and briefly comment on the magnetic flux problem during star formation. Our results are summarized in $\S 5$.

\section{Characteristics of Model Clouds}

\subsection{Pre-PMF Phase}

For the pre-PMF phase of core evolution, which encompasses the formation (due to ambipolar diffusion) and contraction of magnetically supercritical cores in magnetically subcritical clouds, we use the models described in CM93, CM94, and CM95. Further details are given in Appendix A. Here we summarize key features of these models.

Clouds are modeled as axisymmetric thin disks, with half-thickness $Z(r, t)$, embedded 
in a constant-pressure external medium, with the axis of symmetry aligned with the $z$ axis of a cylindrical polar coordinate system $(r, \phi, z)$; exact balance between thermal-pressure and gravitational forces along magnetic field lines is assumed to hold at all times. These simplifying assumptions are based on the results of Fiedler \& Mouschovias (1992, 1993), who found that initially uniform, spherical or cylindrically symmetric, self-gravitating magnetic molecular clouds rapidly flatten and establish force balance (between thermal-pressure and gravitational forces) along magnetic field lines. In their typical models, balance of forces along field lines was maintained even after the onset of dynamical contraction perpendicular to the field lines. The collisional friction of (charged and neutral) grains, which in certain cases can be significant (CM94, CM95), is ignored in this paper. We also neglect the effect of rotation and magnetic braking (e.g., Basu \& Mouschovias 1994, 1995a, b); they will be considered in a later paper.

Model clouds are initially in magnetohydrostatic equilibrium and would remain so indefinitely in the absence of ambipolar diffusion. Hence, any evolution is entirely the result of ambipolar diffusion, which is "turned on" at time $t=0$. The evolution of a magnetically supercritical core is followed up to a time $t_{\text {int }}$, at which the central density exceeds $10^{11} \mathrm{~cm}^{-3}$. At this point the pre-PMF calculation is halted. We then proceed as described in the next subsection.

\subsection{PMF Phase and Subsequent Evolution; Modification of the Ambipolar Diffusion Models}

To calculate this phase of the evolution we use as initial data the values of the physical quantities (column density, magnetic field, neutral infall speed, etc.) of the pre-PMF epoch at time $t_{\text {int }}$. To numerically calculate through the PMF phase of evolution, we use the "central sink cell" method originally employed by Boss \& Black (1982) to model the collapse of nonmagnetic, isothermal clouds. This method was also used in the collapse studies of Foster \& Chevalier (1993) and Tomisaka (1996). In this method we make the central cell of our (stationary) computational mesh a sink cell, with size equal to the mesh inner boundary radius $r=r_{\text {inner }}$ : the central sink cell is essentially a "hole" at the origin of our computational mesh. Use of the central sink cell keeps the numerical time step $\delta t_{\text {num }}$ $\left[\sim r_{\text {inner }} /\left|v_{\mathrm{n}}\left(r_{\text {inner }}\right)\right|\right.$, where $v_{\mathrm{n}}$ is the infall speed of the neutrals] finite throughout the PMF epoch.

We fix the value of $r_{\text {inner }}$ at the radius at which the governing equations of a model cloud no longer remain valid. Two fundamental assumptions of our models are isothermality and the freezing of magnetic flux into the ions. The isothermal approximation breaks down for 
$n_{\mathrm{n}} \gtrsim 10^{11} \mathrm{~cm}^{-3}$ (Gaustad 1963; Hayashi 1966; Larson 1969; Winkler \& Neuman 1980); at densities above this value, radiative heating of the gas can no longer be neglected. Freezing of the magnetic field in the ions is no longer valid when the dimensionless ion magnetic attachment parameter $\Gamma_{\mathrm{i}} \equiv \omega_{\mathrm{i}} \tau_{\mathrm{in}}$, also known as the ion Hall parameter, is $\lesssim 1$ (see eqs. [38] and [39] of CM93), where $\omega_{\mathrm{i}}$ is the ion gyrofrequency and $\tau_{\text {in }}$ is the ion-neutral collisional timescale. [3 The CM94 collapse model that neglects the collisional effect of grains (see their Figs. $2 a$ and $2 f)$ yields the approximate scaling $B_{z \text {,eq }} \simeq 3.5 \times 10^{3}\left(n_{\mathrm{n}} / 2.6 \times 10^{9} \mathrm{~cm}^{-3}\right)^{0.4} \mu \mathrm{G}$ for the equatorial $(z=0)$ magnetic field. Inserting this relation into equation (40) of CM93 gives $\Gamma_{\mathrm{i}} \lesssim 1$ for $n_{\mathrm{n}} \gtrsim 5 \times 10^{10} \mathrm{~cm}^{-3}$. Thus, coincidentally, the assumption of freezing of magnetic flux in the ions breaks down at effectively the same density as the one where the isothermality approximation also becomes invalid. We take $r_{\text {inner }}$ to be the radius at which $n_{\mathrm{n}}\left(r_{\text {inner }}\right) \simeq 10^{11} \mathrm{~cm}^{-3}$. Typically, this corresponds to $r_{\text {inner }} \gtrsim 5$ AU (see $\S 3.3$ ).

Mass and magnetic flux accumulate in the central sink from our active computational region, consisting of all the cells located at $r \geq r_{\text {inner }}$; the rate at which mass is advected into the central sink is

$$
\dot{M}_{\text {cent }} \equiv \frac{\partial M_{\text {cent }}}{\partial t}=-\left.2 \pi r \sigma_{\mathrm{n}} v_{\mathrm{n}}\right|_{r=r_{\text {inner }}},
$$

and the rate at which magnetic flux flows into the central cell is

$$
\dot{\Phi}_{B, \mathrm{cent}} \equiv \frac{\partial \Phi_{B, \mathrm{cent}}}{\partial t}=-\left.2 \pi r B_{z, \mathrm{eq}} v_{\mathrm{i}}\right|_{r=r_{\mathrm{inner}}}
$$

where $M_{\text {cent }}(t)$ and $\Phi_{B \text {,cent }}(t)$ are the central mass and magnetic flux, $\sigma_{\mathrm{n}}(r, t)$ and $B_{z, \text { eq }}(r, t)$ are the column density and magnetic field strength (in the equatorial plane of the disk), and $v_{\mathrm{n}}(r, t)$ and $v_{\mathrm{i}}(r, t)$ are the neutral and ion infall speeds.

Despite the fact that the mass and flux contained within the central sink cell are exterior to the active computational region in our models, they still affect the evolution of the collapse through their gravitational and magnetic influence on the matter at $r \geq r_{\text {inner }}$. This has to be incorporated into the calculation of the radial gravitational and magnetic

\footnotetext{
${ }^{3}$ When $\Gamma_{\mathrm{i}}<1$ the assumption of flux freezing in the ions is no longer valid. However, one can still consider the magnetic flux to be frozen into the electrons, if the electrons are the main charge carriers (as opposed to charged grains) and the electron magnetic attachment parameter $\Gamma_{\mathrm{e}} \equiv \omega_{\mathrm{e}} \tau_{\mathrm{en}}>1$ (for a discussion, see the Appendix of Königl 1989). At even higher densities than we consider here, $\Gamma_{\mathrm{e}}$ becomes $<1$ and the effect of Ohmic dissipation has to be included in the magnetic induction equation (Spitzer 1963; Pneuman \& Mitchell 1965; Norman \& Heyvaerts 1985; Nakano \& Umebayashi 1986a,b). For detailed discussions of the effect of a finite conductivity on the MHD equations for multicomponent plasmas at the high densities encountered in collapsing cores and/or protostellar accretion disks we refer the reader to Nakano (1984), Draine (1986), Königl (1989), Wardle \& Königl (1993), Mouschovias (1996), and Wardle \& Ng (1998).
} 
field components. The radial gravitational acceleration in a thin disk is (see CM93, eqs. [31a] and [31b])

$g_{r}(r)=2 \pi G \frac{d}{d r} \int_{0}^{\infty} d r^{\prime} r^{\prime} \sigma_{\mathrm{n}}\left(r^{\prime}\right) \int_{0}^{\infty} d k J_{0}(k r) J_{0}\left(k r^{\prime}\right)=-2 \pi G \int_{0}^{\infty} d k J_{1}(k r) \int_{0}^{\infty} d r^{\prime} r^{\prime} \sigma_{\mathrm{n}}\left(r^{\prime}\right) J_{0}\left(k r^{\prime}\right)$,

where $J_{0}$ and $J_{1}$ are Bessel's functions of order 0 and 1 , respectively. One can show by direct integration of equation (4) that, for $\sigma_{\mathrm{n}}(r) \propto r^{s_{\sigma}}$, where $s_{\sigma}$ is a constant between $-1 / 2$ (corresponding to free-fall during the post-PMF phase; see $\S 3.3$ ) and -1 (typical of the late stages of the pre-PMF phase, see $\S 3.2), g_{r}(r)=-\mathcal{F}_{\sigma} M(r) / r^{2}$, where $M(r)$ is the mass enclosed within the radius $r$ and $\mathcal{F}_{\sigma}$ is a constant between 0.68 and 1 . As an example, column density profiles $\sigma_{\mathrm{n}}(r) \propto r^{-0.95}$ typically developed in the inner regions of the contracting cores of the models presented by CM94 and CM95 (see also $\S 3.2$ ), corresponding to $\mathcal{F}_{\sigma} \simeq 0.82$ in the above expression. Considering the alternative case of a highly localized source, such as a uniform-density sphere of radius $R_{\star}\left(\ll r_{\text {inner }}\right)$ and mass $M_{\star}$, the column density obeys the relation

$$
\begin{array}{rlrl}
\sigma_{\mathrm{n}}(r) & =\frac{3}{2 \pi} \frac{M_{\star}}{R_{\star}^{2}}\left[1-\left(\frac{r}{R_{\star}}\right)^{2}\right]^{1 / 2}, r \leq R_{\star}, \\
& =0, & r>R_{\star},
\end{array}
$$

and one finds from the solution of equation (1) that, for $r>R_{\star}, g_{r}(r)=-G M_{\star} / r^{2}$, as expected. Hence $g_{r}\left(r>r_{\text {inner }}, t\right) \simeq-G M(r, t) / r^{2}$ for the cases of a power-law column density profile and of a localized mass source. As these two extremes tend to bracket the range of possible behaviors of $\sigma_{\mathrm{n}}(r, t)$ in the innermost flux tubes of the core, we may use the expression

$$
g_{r}(r)=-\frac{G M_{\text {cent }}}{r^{2}}+2 \pi G \frac{d}{d r} \int_{0}^{\infty} d r^{\prime} r^{\prime} \sigma_{\mathrm{n}}\left(r^{\prime}\right) \int_{0}^{\infty} d k J_{0}(k r) J_{0}\left(k r^{\prime}\right)
$$

during the PMF epoch. Extension of the integral in equation (6) to $r=0$ is obtained, with negligible error, by setting $\sigma_{\mathrm{n}}\left(r<r_{\text {inner }}\right)=\sigma_{0}$, where $\sigma_{0}$ is a constant that satisfies the condition $0 \leq \sigma_{0} \leq \sigma_{\mathrm{n}}\left(r_{\text {inner }}\right)$. A description of the numerical method used to solve the integral term on the r.h.s. of equation (6) is given in $\S 2.4$ of Morton, Mouschovias, \& Ciolek (1994, hereafter MMC94).

As shown in $\S 3.1 .1$ of CM93, the $r$ component of the magnetic field at the surface of the disk, which appears in the restoring magnetic tension force (see CM93, eq. [28c]), is given by

$$
\begin{aligned}
B_{r, Z}(r) & =-\frac{d}{d r} \int_{0}^{\infty} d r^{\prime} r^{\prime}\left[B_{z, \mathrm{eq}}\left(r^{\prime}\right)-B_{\mathrm{ref}}\right] \int_{0}^{\infty} d k J_{0}(k r) J_{0}\left(k r^{\prime}\right) \\
& =\int_{0}^{\infty} d k J_{1}(k r) \int_{0}^{\infty} d r^{\prime} r^{\prime}\left[B_{z, \mathrm{eq}}\left(r^{\prime}\right)-B_{\mathrm{ref}}\right] J_{0}\left(k r^{\prime}\right)
\end{aligned}
$$


where the constant $B_{\text {ref }}$ is the external (background) magnetic field strength at $r, z \rightarrow \infty$. Typically, $B_{z, \text { eq }}(r) \gg B_{\text {ref }}$ in the inner flux tubes of a contracting core (CM94, CM95; Basu \& Mouschovias 1994, 1995a, b). f

By analogy with the discussion of the gravitational field $g_{r}(r)$ in the preceding paragraph, calculation of $B_{r, Z}(r)$ for the bracketing source magnetic field profiles $B_{z, \text { eq }}(r) \propto r^{-s_{B}}$, and that of a central point-flux source [i.e., $B_{z, \text { eq }}(r)=B_{\star}=$ const for $r \leq R_{\star}$, and $B_{z, \text { eq }}(r)=0$ for $r>R_{\star}$, equation (7) yields, for both cases, $B_{r, Z}(r) \simeq \Phi_{B}(r) / 2 \pi r^{2}$, where $\Phi_{B}(r)$ is the total magnetic flux enclosed within radius $r$. It follows then, using a derivation similar to that of equation (6), that $B_{r, Z}\left(r>r_{\text {inner }}, t\right)$ after PMF can be written as

$$
B_{r, Z}(r)=\frac{\Phi_{B, \mathrm{cent}}}{2 \pi r^{2}}-\frac{d}{d r} \int_{0}^{\infty} d r^{\prime} r^{\prime}\left[B_{z, \mathrm{eq}}\left(r^{\prime}\right)-B_{\mathrm{ref}}\right] \int_{0}^{\infty} d k J_{0}(k r) J_{0}\left(k r^{\prime}\right) .
$$

Extension of the integral on the r.h.s. of equation (8) to $r<r_{\text {inner }}$ is done in a fashion similar to that discussed above in connection with equation (6).

The effect of the gravitational field of the central point mass also needs to be included in the equation of quasistatic equilibrium along magnetic flux tubes. Assuming balance of gravitational and thermal-pressure forces in the $z$ direction within the disk yields

$$
\rho_{\mathrm{n}} C^{2}=P_{\mathrm{ext}}+\frac{B_{r, Z}^{2}}{8 \pi}\left[1+\left(\frac{\partial Z}{\partial r}\right)^{2}\right]+\frac{\pi}{2} G \sigma_{\mathrm{n}}^{2}+\frac{G M_{\mathrm{cent}} \rho_{\mathrm{n}}}{r}\left\{1-\left[1+\left(\frac{\sigma_{\mathrm{n}}}{2 \rho_{\mathrm{n}} r}\right)^{2}\right]^{-1 / 2}\right\} .
$$

In deriving equation (9) we have used, as in CM93-CM95, the "one-zone approximation" for the relation between the mass density $\rho_{\mathrm{n}}$ and the column density $\sigma_{\mathrm{n}}: \sigma_{\mathrm{n}}(r, t)=\int_{-Z(r, t)}^{Z(r, t)} \rho_{\mathrm{n}}(r, t) d z=2 \rho_{\mathrm{n}}(r, t) Z(r, t)$. The first three terms on the r.h.s. of equation (9), present even in the absence of a central point mass (see CM93, eq. [26]), are, respectively, the constant external pressure, the magnetic squeezing associated with the radial field component at the disk surface, and the self-gravitational stress of the matter contained in a flux tube; the last term on the r.h.s. of equation (9) is the tidal gravitational stress corresponding to the $z$ component of the gravitational field of the central point mass. Equation (9) simply states that the pressure in the equatorial plane is equal to the sum of

\footnotetext{
${ }^{4}$ Note that, under the conditions $B_{z, \text { eq }}(r) \gg B_{\text {ref }}$ and $B_{z, \text { eq }}(r) \propto \sigma_{\mathrm{n}}(r)$, which in our model calculations

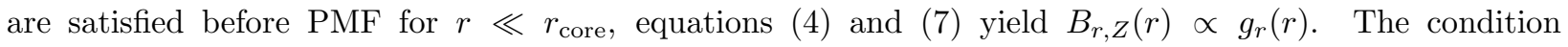
$B_{z, \text { eq }}(r) \propto \sigma_{\mathrm{n}}(r)$ expresses flux freezing into the neutrals, and the relation $B_{r, Z} \propto g_{r}$ was derived under this assumption by Li \& Shu (1997) and Zweibel \& Lovelace (1997), who considered model clouds with $B_{\text {ref }}=0$. This relation was also employed by Basu (1997) in modeling pre-PMF core collapse.
} 
the external thermal plus magnetic stresses and the total gravitational stress acting on each flux tube; this equation is used to calculate $\rho_{\mathrm{n}}(r, t)$. .

As it turns out, the first two terms on the r.h.s. of equation (9) have a negligible effect on the disk evolution in our simulations. In particular, the ratio of $P_{\text {ext }}$ and the self-gravitational stress is generally $\ll 1$ in the inner flux tubes of the core (see eq. [A2]). Furthermore, we have verified that, with the exception of the innermost zones of the active computational grid near the end of our typical simulation, the magnetic squeezing term is smaller than the total gravitational stress in equation (9). Since the properties of these zones have only a small effect on the core structure at that epoch (in particular, the ambipolar diffusion-driven shock that propagates through the core has by that time reached much larger radii; see $§ 3.3$ ), we have, for simplicity, omitted this term altogether in our calculations. As we show in Appendix B, when the core approaches free-fall collapse under these conditions, equation (9) implies $\rho_{\mathrm{n}} \propto r^{-2}$. This behavior, which differs from the dependence $\rho_{\mathrm{n}} \propto r^{-3 / 2}$ that characterizes spherical infall onto a point mass, results from our use of the one-zone approximation in the relation between the column density and mass density for the thin-disk cloud model.

A final modification that is required for the post-PMF phase of collapse is in the ion force equation, from which the ion-neutral drift speed $v_{\mathrm{D}}=v_{\mathrm{i}}-v_{\mathrm{n}}$ is derived (see CM93, eqs. [50] and [51]). This has to do with the fact that, as first pointed out by Mouschovias \& Paleologou (1981), the ion-neutral collision rate $\langle\sigma w\rangle_{\mathrm{ni}}=1.7 \times 10^{-9} \mathrm{~cm}^{3} \mathrm{~s}^{-1}=$ const (used in the ambipolar diffusion models of CM93-CM95), which was derived by using the well-known Langevin approximation (see, e.g., Gioumousis \& Stevenson 1958; McDaniel \& Mason 1973), is valid only so long as the drift speed satisfies the criterion $\langle\sigma w\rangle_{\mathrm{ni}} /\left|v_{\mathrm{D}}\right| \geq \sigma_{\mathrm{geo}}$, where $\sigma_{\text {geo }}$ is the geometric cross section for ion- $\mathrm{H}_{2}$ collisions $\left(=\pi\left[a_{\text {ion }}+a_{\mathrm{H}_{2}}^{2}\right.\right.$, where $a_{\text {ion }}$ and $a_{\mathrm{H}_{2}}$ are the ion and $\mathrm{H}_{2}$-molecule radii, respectively). Therefore, for drift speeds $v_{\mathrm{D}}>v_{\mathrm{D}, \text { crit }}=\langle\sigma w\rangle_{\mathrm{ni}} / \sigma_{\text {geo }}$, the quantity $\sigma_{\text {geo }}\left|v_{\mathrm{D}}\right|$ must be used for the collision rate, and

\footnotetext{
${ }^{5}$ For computational convenience, we continue to use the quasistatic approximation for motions in the $z$ direction even when there is dynamical collapse in the $r$ direction. To test the validity of this assumption, we have also run models that did not employ the quasistatic assumption along flux tubes and instead allowed for acceleration of the neutrals in the $z$ direction. In these models there was initially a short-lived phase of vertical dynamical relaxation and oscillation in the central flux tubes, followed by rapid reestablishment of balance of forces along flux tubes, in agreement with equation (9). The overall evolution of these models differed only slightly from those in which balance of vertical forces was assumed at all times. However, the computing time needed to run models that did not assume vertical force equilibrium was typically an order of magnitude longer than for models that always used the quasistatic approximation.
} 
instead of using equation (51) of CM93, the appropriate equation for the drift speed is

$$
v_{\mathrm{D}}(r, t)=\left\{1.4\left[1+0.067 \frac{\left(m_{\mathrm{H}_{2}} / 2 \text { a.m.u. }\right)}{\left(m_{\mathrm{i}} / 30 \text { a.m.u. }\right)}\right] \frac{F_{\mathrm{mag}, \mathrm{r}}(r, t)}{\sigma_{\text {geo }} n_{\mathrm{i}}(r, t) \sigma_{\mathrm{n}}(r, t)}\right\}^{1 / 2},
$$

where $F_{\text {mag,r }}(r, t)$ is the total (radial) magnetic force per unit area, which includes both the magnetic tension and pressure stresses (see eq. [28c] of CM93). The factor 1.4 in equation (10) reflects the fact that we are neglecting the inertial effect of a $20 \% \mathrm{He}$ abundance in the neutral-ion collision timescale $\tau_{\text {ni }}$ (see eq. [1]). For the various species of ions we include in our models (see Appendix A) we adopt a "generic" ion radius of $0.8 \AA$; this yields a critical drift speed $v_{\mathrm{D} \text {,rrit }}=12 \mathrm{~km} \mathrm{~s}^{-1}$, only slightly different from the value of $10 \mathrm{~km} \mathrm{~s}^{-1}$ obtained by Mouschovias \& Paleologou (1981), who had assumed a plasma consisting solely of $\mathrm{Na}^{+}$ions. Hence, when $v_{\mathrm{D}}(r, t)>v_{\mathrm{D}, \text { crit }}$, we use equation (10); otherwise, equation (51) of CM93 is used. As we show in $\S 3.3$, ambipolar diffusion can continue to be effective even in dynamically contracting cores, and drift speeds $v_{\mathrm{D}}>v_{\mathrm{D}, \text { crit }}$ may be attained in certain core regions during the post-PMF epoch.

Spatial discretization and time integration of the equations governing the evolution of a model cloud are carried out by the methods described in MMC94 - with the exception that spatial derivatives in the first computational cell, with inner boundary $r=r_{\text {inner }}$, are calculated by using one-sided differences instead of the three-point technique described in $\S$ A2.4 of Mouschovias \& Morton (1991). All computations were performed on an SGI R-4000 Indigo workstation; running in background, a typical model calculation took $\sim 2$ weeks to form a central protostar with a mass of $1 M_{\odot}$.

As we show in $\S 3.3$, ambipolar diffusion significantly alters the evolution of a collapsing core following PMF. To verify that the results of our typical simulation represent a real effect that arises from a physical diffusion process and are not an artifact of numerical diffusivity, we have run a parallel model that corresponds to flux freezing into the neutrals for all times $\Delta t \geq 0$. (This was accomplished simply by setting $v_{\mathrm{i}}=v_{\mathrm{n}}$ in our numerical code.) The results of this calculation (detailed in $\S 3.3$ ) have revealed no evidence of numerical diffusion arising from our finite discretization scheme: each neutral fluid element was found to evolve with constant mass and magnetic flux throughout the entire simulation, as would be expected for a fluid with a frozen-in magnetic field. In $\S 3.3$ we therefore use the results of the frozen-flux cloud calculation as an aid in isolating and interpreting the effects of ambipolar diffusion in our typical model (see also Appendix C). We note in this connection that we have also run other ambipolar-diffusion models with different mesh sizes and core resolutions and obtained essentially the same results as in our typical model. This further confirms the absence of numerical-diffusion effects in our simulations. In addition, we have tested the effect of varying the assumed profile of $B_{z, \text { eq }}$ for $r<r_{\text {inner }}$ in calculating 
$B_{r, Z}$ from equation (8), and confirmed that our quantitative results were insensitive to the detailed form of that profile.

\section{Numerical Simulation}

\subsection{Model Parameters}

We present a typical model of the dynamical evolution of a magnetically supercritical core through the PMF phase. The values of the dimensionless parameters of this model are listed in Table 1 and Table 2. They are similar to that of model $\mathrm{A}_{\mathrm{UV}}$ presented in CM95. The values of the free parameters selected could be used to represent a molecular cloud with temperature $T=10 \mathrm{~K}$, radius $R_{0}=4.29 \mathrm{pc}$, and total mass $M_{\mathrm{d}}=98.3 M_{\odot}$. For a gas of $\mathrm{H}_{2}$ with a $20 \% \mathrm{He}$ abundance the mean mass of a neutral particle is $m_{\mathrm{n}}=2.33$ a.m.u. and the isothermal speed of sound is $C=\left(k_{\mathrm{B}} T / m_{\mathrm{n}}\right)^{1 / 2}=0.19 \mathrm{~km} \mathrm{~s}^{-1}$. The initial central density $n_{\mathrm{n}, \mathrm{c} 0}$, column density $\sigma_{\mathrm{n}, \mathrm{c} 0}$, and magnetic field strength $B_{z, \mathrm{eq}, \mathrm{c} 0}$ are $2.6 \times 10^{3} \mathrm{~cm}^{-3}$, $5.59 \times 10^{-3} \mathrm{~g} \mathrm{~cm}^{-2}$, and $35.3 \mu \mathrm{G}$, respectively. The dimensional values of the parameters used in the calculation of the equilibrium abundances of charged particles, such as the abundances of the different atomic species, cosmic-ray and UV ionization rates, chemical and charge-transfer reaction rates, etc., are the same as those cited in $\S 3.1$ of CM95, with the exception that the probability $\mathcal{P}_{\mathrm{i}}$ of ions sticking onto grains (see CM93, eqs. [57b] and [57c]) has been changed from 0.9 to 0.99 . For the purposes of calculating ion abundances, we assume a uniform population of grains with radius $a=3.75 \times 10^{-6} \mathrm{~cm}$. .

\subsection{Pre-PMF Phase: Supercritical Core Formation and Contraction}

Models of the self-initiated (due to ambipolar diffusion) formation and contraction of magnetically supercritical cores have been presented and discussed at length by Fiedler \& Mouschovias (1993), CM94 and CM95, and Basu \& Mouschovias (1994, 1995a, b); we refer the reader to these papers for more detailed descriptions of the physics of the formation of protostellar cores in magnetically supported interstellar clouds. During the pre-PMF phase, the evolution of the core of the typical model cloud presented here is similar to that

\footnotetext{
${ }^{6}$ Although we do not account for grain-neutral friction in this model, our results would be little changed if we had instead used grains with radius $a \gtrsim 7 \times 10^{-6} \mathrm{~cm}$ and included the effect of grain-neutral collisions, as grains of this size or larger contribute only marginally to the total collisional force on the neutrals (see model 2 of Ciolek 1993).
} 
of model $\mathrm{A}_{\mathrm{UV}}$ discussed at length in $\S 3.2 .1$ of CM95; for brevity, we summarize here only some of the features of the core in the pre-PMF phase.

Figures $1 a-1 i$ display, respectively, the density (normalized to $n_{\mathrm{n}, \mathrm{c} 0}$ ), column density (normalized to $\sigma_{\mathrm{n}, \mathrm{c} 0}$ ), mass-to-flux ratio (in units of the critical value for collapse), magnetic field strength in the equatorial plane of the disk (normalized to $B_{z, \text { eq,c0 }}$ ), $r$ component of the magnetic field at the surface of the disk (normalized to $B_{z, \mathrm{eq}, \mathrm{c} 0}$ ), infall speed of the neutrals (normalized to $C$ ), drift speed (normalized to $C$ ), mass infall rate $\dot{M}$ (in units of $\left.M_{\odot} \mathrm{Myr}^{-1}=0.65 C^{3} / G\right)$, and ratio of local cloud vertical half-thickness $Z$ and radius $r$ as functions of $r / R_{0}$, at eleven different times $t_{j}$. The times $t_{j}$ correspond to when the central density $n_{\mathrm{n}, \mathrm{c}}\left(t_{j}\right)=10^{j} n_{\mathrm{n}, \mathrm{c} 0}$; dimensionally, these times are $t_{0}=0, t_{1}=7.56 \mathrm{Myr}$, $t_{2}=9.29 \mathrm{Myr}, t_{3}=9.56 \mathrm{Myr}, t_{4}=9.607 \mathrm{Myr}, t_{5}=9.6167 \mathrm{Myr}, t_{6}=9.6169 \mathrm{Myr}$, $t_{7}=9.6190 \mathrm{Myr}, t_{8}=9.61977 \mathrm{Myr}, t_{9}=9.61981 \mathrm{Myr}$, and $t_{10}=9.61983 \mathrm{Myr}$. An asterisk on a curve in Figure 1 locates the instantaneous radius $R_{\text {crit }}\left(t_{j}\right)$ inside which the total-mass to total-flux ratio is equal to the critical value for collapse, i.e.,

$$
\left(\frac{M}{\Phi_{B}}\right)_{r=R_{\mathrm{crit}}\left(t_{j}\right)}=\frac{1}{2 \pi G^{1 / 2}} .
$$

An open circle locates the instantaneous critical thermal $\left(\simeq\right.$ Jeans) lengthscale $\lambda_{\mathrm{T}, \mathrm{crit}}\left(t_{j}\right)$ [ $=C^{2} / 2 G \sigma_{\mathrm{n}, \mathrm{c}}\left(t_{j}\right)$, where $\sigma_{\mathrm{n}, \mathrm{c}}\left(t_{j}\right)$ is the central column density at time $t_{j}$; Mouschovias 1991].

During the later stages of the evolution $\left(t z t_{1}\right)$, the core develops a uniform central region [of radius $\simeq \lambda_{\mathrm{T} \text {, crit }}\left(t_{j}\right)$ ], which is continually shrinking in both size and mass, and a "tail" of matter and magnetic field left behind by the central region. Inside the "tail" region, near power-law behavior emerges, with $d \ln \rho_{\mathrm{n}} / d \ln r \approx-2, d \ln \sigma_{\mathrm{n}} / d \ln r \approx-1$, and $d \ln B_{z, \text { eq }} / d \ln r \approx-1$ deep inside the core, and with $d \ln \rho_{\mathrm{n}} / d \ln r \approx-1.5$, $d \ln \sigma_{\mathrm{n}} / d \ln r \approx-0.7$, and $d \ln B_{z, \mathrm{eq}} / d \ln r \approx-0.6$ further out, where most of the core mass is contained. As noted by Basu (1997), for $t \gtrsim t_{1}$, the column density and $z$ component of the magnetic field within the inner part of the core $\left(r \ll R_{\text {crit }}\right)$ are well approximated by the relations

$$
\sigma_{\mathrm{n}}(r, t) \simeq \frac{\sigma_{\mathrm{n}, \mathrm{c}}(t)}{\left(1+\left[r / 2 \lambda_{\mathrm{T}, \mathrm{cr}}(t)\right]^{2}\right)^{1 / 2}}
$$

and

$$
B_{z, \mathrm{eq}}(r, t) \simeq \frac{B_{z, \mathrm{eq}, \mathrm{c}}(t)}{\left(1+\left[r / 2 \lambda_{\mathrm{T}, \mathrm{cr}}(t)\right]^{2}\right)^{1 / 2}},
$$

where $\sigma_{\mathrm{n}, \mathrm{c}}(t)$ and $B_{z, \mathrm{eq}, \mathrm{c}}(t)$ are the values of $\sigma_{\mathrm{n}}$ and $B_{z, \text { eq }}$ in the uniform central region at time $t$ (see Figs. $1 b$ and $1 d$ ). PMF occurs when $\lambda_{\mathrm{T}, \mathrm{cr}}(t) \rightarrow 0$. In the inner core the magnitude of the retarding magnetic force decreases relative to the gravitational force, 
becoming comparable to that of the thermal-pressure force. Further out, at radii $\sim R_{\text {crit }}\left(t_{j}\right)$, the magnetic force is the primary source of support against self-gravity.

By the time $t_{10}$ the core has a radius $R_{\text {crit }}\left(t_{10}\right)=2.59 \times 10^{-2} R_{0}=0.111 \mathrm{pc}$ and a mass $M_{\text {core }}=5.37 M_{\odot}$. These values are in excellent agreement with typical observations of dense ammonia cores in star-forming molecular clouds (e.g., Ladd, Myers, \& Goodman 1994). Separation of the magnetically supercritical core from the massive, subcritical envelope is demonstrated in Figure $1 h$, which shows that the mass infall rate $\dot{M}$ is reduced by more than four orders of magnitude for $r>R_{\text {crit }}\left(t_{j}\right)$. This is due to the effective freezing of the magnetic field in the neutrals at large radii, which is a consequence of the comparatively high degree of ionization brought about by the penetration of the external UV radiation field into the optically thin cloud envelope (see CM95 for a discussion). Ø

We note the following results of interest for the pre-PMF phase of evolution. First, as can be seen from Figures $1 f$ and $1 g$, the magnitude of the infall speed of the neutrals in the inner regions of the collapsing core is comparable to the isothermal speed of sound $C$ (and thus also to the fast-magnetosonic speed $v_{\mathrm{ms}}=\left[C^{2}+v_{\mathrm{A}}^{2}\right]^{1 / 2}$, where $v_{\mathrm{A}}=B_{z, \mathrm{eq}} /\left[4 \pi \rho_{\mathrm{n}}\right]^{1 / 2}$ is the Alfvén speed, since $v_{\mathrm{ms}}$ is nearly equal to $C$ in these regions). Moreover, at time $t_{10}$, the inward acceleration $a_{\mathrm{n}}$ of the neutrals lies in the range $0.25 g_{r}-0.5 g_{r}$ in the inner regions of the core, and $\left|v_{\mathrm{n}}\right|$ is a fraction $\sim 0.55$ of the free-fall speed $\left(\simeq 1.98 C\right.$ ) at $r=r_{\text {inner }}$ (see $\S 3.3$ ). Hence the core is indeed dynamically collapsing (although not freely falling), so, as we noted in $\S 1$, treating a supercritical core as being in near hydrostatic equilibrium at PMF is not a valid approximation for molecular cloud cores that form and evolve by ambipolar diffusion.

Second, we note the increase of the drift speed $v_{\mathrm{D}}$ in the inner flux tubes for $t \gtrsim t_{6}$ (see Fig. 1h), which indicates that ambipolar diffusion can continue to operate even during the dynamical collapse of the core. This is primarily due to the phenomenon of ion depletion, in which ions become increasingly attached onto grains at higher densities, thereby reducing the relative abundance of ions in the gas phase. This, in turn, results in a higher diffusion rate (see CM93, CM94) and leads to an increase (for $t>t_{6}$ ) of the mass-to-flux ratio in the flux tubes that thread the inner core (see Fig. 1c). (The effect of ambipolar diffusion on the mass-to-flux ratio during the approach to PMF is also discussed in Basu 1997.) As we show in the next subsection (see also $\S 1$ ), ambipolar diffusion in the interior flux tubes is

\footnotetext{
${ }^{7}$ The outer envelope may be supported even better than we have calculated in view of the fact that the UV photoionization rates could be higher than those listed in Table 2, in which case the fractional ionization values in the outer regions of the cloud (corresponding to visual extinctions $\lesssim 4$ ) would be slightly larger (Ruffle et al. 1998).
} 
increased dramatically after PMF and plays a significant dynamical role in the subsequent evolution of the core.

Finally, we notice that by the time $t_{10}, Z / r<1$ throughout the whole cloud, except for a very small inner region of the core $\left(r / R_{0} \lesssim 10^{-7}\right.$; see Fig. $\left.1 i\right)$. Note that $Z / r>1$ does not necessarily indicate an inconsistency in our use of the thin-disk approximation to model clouds. As discussed in CM93 and CM95, this approximation will be valid so long as any scalar quantity $f(r)$ (such as $n_{\mathrm{n}}, \sigma_{\mathrm{n}}, B_{z \text {,eq }}$, etc.) satisfies the condition $f(r, t) /|\partial f / \partial r| \geq Z(r, t)$. Examination of Figures $1 a, 1 b$, and $1 d$ reveals that this condition is satisfied even in the uniform-density central region because $\partial f / \partial r \approx 0$ for $r \rightarrow 0$ before PMF. As it turns out, the innermost region of the core, which at later times happens to contain densities $n_{\mathrm{n}}>10^{11} \mathrm{~cm}^{-3}$ (see Fig. 1a), is in any case excluded from our calculation of the post-PMF core evolution for the reasons outlined in $\S 2.2$.

\subsection{Point-Mass Formation and Protostellar Accretion Phase of Core Evolution}

The physical data corresponding to the model at time $t_{\text {int }}=t_{10}$ are used as input for the protostellar-accretion phase of the calculation. (Our results for the post-PMF epoch are relatively insensitive to the particular value of $t_{\text {int }}$ we choose; for instance, we find that the resulting evolution differs only marginally if we use $t_{\text {int }}=t_{8}$ instead.) The boundary of the central sink cell is taken to be $r_{\text {inner }}=8.24 \times 10^{-6} R_{0}=7.31 \mathrm{AU}$. (The post-PMF evolution is also relatively insensitive to the value of $r_{\text {inner }}$ so long as $r_{\text {inner }}$ is much smaller than the core radius $R_{\text {crit }} \approx 0.1 \mathrm{pc}$; for instance, the results for $r_{\text {inner }} \simeq 20 \mathrm{AU}$ are very similar to those of our typical run.) The density at this position and time is $5.0 \times 10^{10} \mathrm{~cm}^{-3}$ (see Fig. $1 a)$, whereas the mass contained in the central cell at this time is $M_{\text {cent }}\left(t_{10}\right)=8.5 \times 10^{-4} M_{\odot}$, which is negligible in comparison with the total core mass $M_{\text {core }}=5.37 M_{\odot}$. Similarly, $\Phi_{B, \text { cent }}\left(t_{10}\right)=6.72 \times 10^{26} \mathrm{Mx}$, which is several orders of magnitude smaller than the total core magnetic flux $\Phi_{B, \text { core }}=1.72 \times 10^{31} \mathrm{Mx}$.

The evolution of the mass in the central sink cell is shown in Figure $2 a$ as a function of $\Delta t=t-t_{10}$. Point-mass formation, signaled by rapid growth of $M_{\text {cent }}$ with time, takes place at $\Delta t \leq 200 \mathrm{yr}$. 1 - because of the nonzero value of $r_{\text {inner }}$, we can only place an upper limit on this time. We terminate the simulation at $\Delta t=1.53 \times 10^{5} \mathrm{yr}$, when $M_{\text {cent }}=1 M_{\odot}$.

\footnotetext{
${ }^{8}$ Therefore, for $\Delta t \gtrsim 10^{3} \mathrm{yr}$, the quantity $\Delta t$ can be considered to be the time elapsed since PMF. This makes our time unit $\Delta t$ the same as the time $t$ typically used in previously published self-similar collapse models (see references cited in $\S 1$ ), which set $t=0$ at PMF.
} 
The accretion rate $\dot{M}_{\text {cent }}$ onto the protostar (in units of $M_{\odot} \mathrm{Myr}^{-1}$ ) is shown in Figure $2 b$ as a function of $\Delta t$ (solid curve). As in the previous (nonmagnetic and magnetic) simulations of dynamical collapse cited in $\S 1, \dot{M}_{\text {cent }}$ rapidly increases imediately after PMF due to the enhancement of the gravitational acceleration brought about by the appearance of the protostar. $\dot{M}_{\text {cent }}$ reaches a maximum value $\simeq 9.4 M_{\odot} \mathrm{Myr}^{-1}$, which is less than the theoretical upper limit of $13 C^{3} / G=20 M_{\odot} \mathrm{Myr}^{-1}$ estimated by Basu (1997) for collapsing cores in which ambipolar diffusion remains operative. $\dot{M}_{\text {cent }}$ decreases for $\Delta t \gtrsim 4 \times 10^{3} \mathrm{yr}$. As we show below, the accretion rate during this time is strongly affected by an ambipolar diffusion-induced hydromagnetic shock that propagates outward and slows the infall of matter at larger radii. We also plot in Figure $2 b$ the central accretion rate of a collapse model (dashed curve) that began with the same initial data as our typical model, but with $v_{\mathrm{D}}$ set equal to zero for $\Delta t \geq 0$ (i.e., for this model, as discussed in $\S 2.2$, the magnetic flux was artificially forced to remain frozen into the neutrals during the post-PMF epoch). Finally, in Figure $2 c$, we plot $\dot{M}_{\text {cent }}$ (in the same units as in Fig. $2 b$ ) as a function of $M_{\text {cent }} / M_{\odot}$. The dashed curve again displays the accretion rate for the frozen-flux model.

Comparing the two models shown in Figures $2 b$ and $2 c$, we note that $\dot{M}_{\text {cent }}$ is the same for $\Delta t<10^{3} \mathrm{yr}$ : this has to do with the fact that the ambipolar diffusion timescale $\tau_{\mathrm{AD}}$ in the typical model is much longer than the gravitational contraction timescale $\tau_{\text {gr }}$ during this period (see Figs. $3 a$ and $3 b$ ), so that diffusion does not greatly alter the evolution. However, for $\Delta t>10^{3} \mathrm{yr}, \tau_{\mathrm{AD}} \simeq \tau_{\mathrm{gr}}$, so ambipolar diffusion is much more effective and leads to a redistribution of the mass in the inner flux tubes of the core (see below). This, in turn, results in a reduction of the mass accretion rate onto the central protostar. Specifically, by the end of the simulation, $\dot{M}_{\text {cent }}$ in the ambipolar-diffusion model is $\simeq 60 \%$ of the accretion rate in the frozen-flux case. The ambipolar diffusion-induced decrease in $\dot{M}_{\text {cent }}$ comes on top of the monotonic decline exhibited in the flux-frozen case for $\Delta t \gtrsim 10^{4} \mathrm{yr}$. The behavior of the frozen-flux model is consistent with the results of other nonmagnetic and magnetic collapse calculations (e.g., Hunter 1977; Foster \& Chevalier 1993; Tomisaka 1996; Safier et al. 1997; Li 1998). As discussed by Basu (1997, § 5), the post-PMF decline in $\dot{M}_{\text {cent }}$ is due to the fact that, at the time of PMF, the outer mass shells of the core are not as strongly accelerated inward (and are therefore moving more slowly than the inner mass shells) because of their much larger initial distance from the central point mass. The maximum values of $\dot{M}_{\text {cent }}$ in both of these numerical models agree with those found in the self-similar magnetic collapse solutions presented in Contopoulos, Ciolek, \& Königl (1997, hereafter referred to as CCK97).

We now show that, during the post-PMF epoch, ambipolar diffusion operates effectively in the inner core even as it continues to undergo dynamical collapse. As we did in $\S 1$, we again examine the ambipolar diffusion timescale $\tau_{\mathrm{AD}}=r / v_{\mathrm{D}}$, which we now express in 
a manner similar to that of Mouschovias $(1989,1991)$. The force (per unit area) on the neutrals is

$$
\sigma_{\mathrm{n}} a_{\mathrm{n}}=\sigma_{\mathrm{n}} g_{r}-C^{2} \frac{\partial \sigma_{\mathrm{n}}}{\partial r}+\frac{\sigma_{\mathrm{n}}}{\tau_{\mathrm{ni}}} v_{\mathrm{D}},
$$

where $a_{\mathrm{n}}=d v_{\mathrm{n}} / d t$ is the acceleration of the neutrals. The first term on the r.h.s. of equation (14) is the gravitational force (including both self-gravity of the gas and the gravitational field of a central point mass), the second term is the thermal-pressure force, and the last term is the frictional force due to collisions between neutrals and ions. Dividing this equation by $r$, one obtains

$$
\frac{\tau_{\mathrm{AD}}}{\tau_{\mathrm{gr}}}=\frac{\left(\tau_{\mathrm{gr}} / \tau_{\mathrm{ni}}\right)}{\left[1-\left(\tau_{\mathrm{gr}} / \tau_{\mathrm{acc}}\right)^{2}-\left(\tau_{\mathrm{gr}} / \tau_{\mathrm{s}}\right)^{2}\right]},
$$

where $\tau_{\mathrm{gr}}\left(\equiv\left[r /\left|g_{r}\right|\right]^{1 / 2}\right)$ is the gravitational contraction timescale (referred to as the dynamical timescale in CM94 and CM95), $\tau_{\text {acc }}\left(\equiv\left[r /\left|a_{\mathrm{n}}\right|\right]^{1 / 2}\right)$ is the acceleration timescale, and $\tau_{\mathrm{s}}\left(\equiv\left[\left(C^{2} / r\right)\left|\partial \ln \sigma_{\mathrm{n}} / \partial r\right|\right]^{-1 / 2}\right)$ is the sound crossing time. Equation (15) is the same as equations (19) and (20) of Mouschovias (1989), except that we use the gravitational contraction timescale $\tau_{\text {gr }}$ instead of the free-fall time $\tau_{\text {ff }}$. Now, during the later stages of dynamical collapse, the ratio $\tau_{\mathrm{gr}} / \tau_{\mathrm{acc}}$ in the denominator approaches unity and tends to increase $\tau_{\mathrm{AD}} / \tau_{\mathrm{gr}}$ (the ratio $\left[\tau_{\mathrm{gr}} / \tau_{\mathrm{s}}\right]^{2}$ is negligible). However, effective diffusion (corresponding to $\tau_{\mathrm{AD}} / \tau_{\mathrm{gr}} \lesssim 1$ ) can still occur if the ratio $\tau_{\mathrm{gr}} / \tau_{\mathrm{ni}}$ in the numerator of equation (15) is small during this period - due either to a decrease in $\tau_{\text {gr }}$ or to an increase in $\tau_{\text {ni }}$. Hence, if $\tau_{\text {gr }} / \tau_{\text {ni }} \ll 1$, ambipolar diffusion can still redistribute mass and flux in a dynamically collapsing core. P That this is indeed the case in our collapse model can be seen in Figures $3 a$ and $3 b$, which show, as functions of $\Delta t, \tau_{\mathrm{AD}} / \tau_{\mathrm{gr}}, \tau_{\mathrm{gr}} / \tau_{\mathrm{ni}}$, and the total (nondimensional) magnetic flux contained within cells 1 and 5 of our computational mesh. Examination of these figures reveals that $\tau_{\mathrm{gr}} / \tau_{\mathrm{ni}}$ decreases with increasing $\Delta t$. This is caused solely by the decrease in the value of $\tau_{\mathrm{gr}} \propto\left|g_{r}\right|^{-1 / 2} \propto M_{\text {cent }}^{-1 / 2}$ (see eq. [6]). On the other hand, $\tau_{\mathrm{ni}} \propto n_{\mathrm{i}}^{-1}$ (see eq. [1]) changes very little during this period due to the fact that, as noted in $\S 3.2$, $n_{\mathrm{i}} \simeq$ const for $n_{\mathrm{n}} \gg 10^{7} \mathrm{~cm}^{-3}$. The rapid increase of $M_{\text {cent }}$ with $\Delta t$ following PMF (see Fig. $2 a$ ) thus leads to a strong decrease in $\tau_{\mathrm{gr}} / \tau_{\mathrm{ni}}$, resulting in a "revitalization" of ambipolar diffusion in the inner flux tubes during this phase. For $\Delta t \gtrsim 10^{3} \mathrm{yr}, \tau_{\mathrm{AD}} / \tau_{\mathrm{gr}} \simeq 1$, and the growth of the magnetic flux contained within these two cells is halted. The flux then begins to "pile up" in the inner cells of the computational mesh. (The revitalization of ambipolar

\footnotetext{
${ }^{9}$ The criterion $\tau_{\mathrm{gr}} / \tau_{\mathrm{ni}} \ll 1$ is equivalent to requiring $\lambda_{\mathrm{M}, \mathrm{cr}} / \lambda_{\mathrm{A}} \ll 1$, where $\lambda_{\mathrm{M}, \mathrm{cr}} \approx v_{\mathrm{A}} \tau_{\mathrm{ff}} \approx v_{\mathrm{A}} \tau_{\mathrm{gr}}$ is the critical magnetic lengthscale, and $\lambda_{\mathrm{A}} \approx v_{\mathrm{A}} \tau_{\text {ni }}$ is the Alfvén lengthscale, as defined by Mouschovias (1991). The condition $\lambda_{\mathrm{M}, \mathrm{cr}} \ll \lambda_{\mathrm{A}}$ for effective ambipolar diffusion during rapid core collapse was put forth by Mouschovias (ib., $\S 4.1$ ).
} 
diffusion after PMF can also be understood in terms of the scaling of the magnetic field following point mass formation; see Appendix C.)

The effect that the piled-up magnetic flux has on the inner flux tubes of the typical model can be seen in Figures $4 a$ and $4 b$, which display, respectively, $B_{z, \text { eq }} / B_{z, \text { eq,c0 }}$ and $\sigma_{\mathrm{n}} / \sigma_{\mathrm{n}, \mathrm{c} 0}$ as functions of $\Delta t$ for cells $2,5,10,15,20,25$, and 27 of the computational mesh. Figure $4 a$ shows that the piling up of magnetic flux begins close to the axis of symmetry and then, with increasing time, becomes noticeable at progressively larger radii. Hence, after PMF, ambipolar diffusion causes a front of magnetic flux to move outward from the innermost flux tubes. Ambipolar diffusion is so effective behind this hydromagnetic disturbance (HMD) that the inward advection of magnetic flux stops — with the field lines being held in place or else moving slowly outward, in either case with $\left|v_{\mathrm{i}}\right| \ll\left|v_{\mathrm{n}}\right|$. This behavior is reproduced by the self-similar collapse solutions of CCK97, which also incorporate the effect of ambipolar diffusion on the post-PMF core evolution. The magnetic field strength $B_{z \text {,eq }}$ is greatly enhanced after the passage of the HMD in each cell. The effect of the HMD on the behavior of the column density varies with the distance from the center. In particular, the column density in cells 2 and 5 shows no effect from the passage of the magnetic field front (see Figs. $4 a$ and $4 b$ ). This is because ambipolar diffusion is so rapid in these cells that very little of the increased magnetic force from the enhanced field is transmitted to the neutrals through ion-neutral collisions. However, after the HMD reaches larger distances, the collisional coupling between the two fluids increases (see Fig. 6h). Furthermore, the piling-up of flux increases the local field strength at these distances by a larger relative factor. As a result of these two effects, the increased magnetic force acting on the neutrals is able to temporarily decelerate the infalling matter (from slightly supersonic to subsonic infall speeds; see Fig. 6e) for cells with index $l \geq 8$. For these cells, $\sigma_{\mathrm{n}}$ increases immediately after the passage of the front. The effect of the HMD at larger radii can be described in terms of a hydromagnetic shock that propagates in the weakly ionized gas. (As noted in $\S 1$, the formation of such a shock was predicted by Li \& McKee 1996; we further analyze the nature of this C-type shock in $\S 4.2$.) After traversing the shock, the neutrals are gradually reaccelerated and eventually reach free-fall speeds. ¿From Figure $4 b$ we find that $s_{\Delta t} \equiv d \ln \sigma_{\mathrm{n}} / d \ln \Delta t$ lies in the range $-0.76 \lesssim s_{\Delta t} \lesssim-0.55$, which can be compared with the value $s_{\Delta t}=-1 / 2$ that characterizes self-similar collapse models of thin, axisymmetric disks (Li \& Shu 1997; CCK97).

The position of the hydromagnetic disturbance $r_{\text {HмD }}(t)$ in units of the cloud radius $R_{0}$ is presented in Figure $5 a$ as a function of $\Delta t$. The speed $v_{\text {HмD }}\left(=d r_{\text {HмD }} / d t\right.$, in units of $\left.C\right)$ of the front in the reference frame of the central protostar is shown in Figure $5 b$. (The time intervals between successive data points in these figures are larger than those in Figures $2-4$. Figures $5 a$ and $5 b$ therefore show a time-averaged motion of the HMD and do not 
exhibit the steady, shock-induced oscillations seen in Figures 2-4 at later times.) Early on, the radially outward motion of the front is nonsteady, and depends on the rate at which the flux external to the front enters and piles up in the region of rapid ambipolar diffusion. At later times, $v_{\text {нмд }}<C$. The Mach number of the hydromagnetic disturbance in the reference frame of the upstream gas, $\mathcal{M}_{\mathrm{HMD}}=\left(v_{\mathrm{HMD}}-v_{\mathrm{n}, \mathrm{u}}\right) / C$ (where $v_{\mathrm{n}, \mathrm{u}}$ is the infall speed of the neutrals just ahead of the front), is shown in Figure $5 c$ as a function of $\Delta t$. The motion of the HMD relative to the upstream gas is supersonic, and, for $\Delta t \gtrsim 10^{4} \mathrm{yr}, \mathcal{M}_{\mathrm{HMD}} \simeq 2.8$. It is also of interest to note that, in the vicinity of the front, the fast-magnetosonic speed $v_{\mathrm{ms}}$ is typically $\lesssim 2 C$. Hence the hydromagnetic Mach number $\mathcal{M}_{\mathrm{ms}, \mathrm{HMD}}=\left(v_{\mathrm{HMD}}-v_{\mathrm{n}}\right) / v_{\mathrm{ms}}$ of the HMD relative to the upstream neutrals is also $>1$.

Spatial profiles of various quantities in the typical model are shown in Figures $6 a-6 i$ as functions of $r / R_{0}$, at seven different times $\Delta t$. As noted earlier, $\Delta t$ is essentially the time elapsed since PMF. For this typical model, $\Delta t_{0}=0, \Delta t_{1}=2.17 \times 10^{2} \mathrm{yr}$, $\Delta t_{2}=6.11 \times 10^{2} \mathrm{yr}, \Delta t_{3}=1.54 \times 10^{3} \mathrm{yr}, \Delta t_{4}=3.81 \times 10^{3} \mathrm{yr}, \Delta t_{5}=2.38 \times 10^{4} \mathrm{yr}$, and $\Delta t_{6}=1.48 \times 10^{5} \mathrm{yr}$. An asterisk on a curve locates, as in Figures $1 a-1 i$, the instantaneous position of the critical flux tube $R_{\text {crit }}\left(\Delta t_{j}\right)$. The core radius does not change from its initial value of $R_{\text {crit }}(\Delta t=0)=R_{\text {crit }}\left(t_{10}\right)=2.59 \times 10^{-2} R_{0}=0.111 \mathrm{pc}=2.30 \times 10^{4} \mathrm{AU}$, providing further evidence for effective core-envelope separation, as discussed in earlier models presented by Mouschovias and coworkers (see references in $\S 1$ ).

Figure $6 a$ shows the total mass $M$ in $M_{\odot}$. For $\Delta t_{j}>0$ the curves flatten for small radii, revealing point-mass formation since $M(r) / r \neq 0$ for $r \rightarrow 0$. For $r>R_{\text {crit }}\left(\Delta t_{j}\right), M(r)$ changes very little as the magnetically subcritical envelope continues to be supported by magnetic forces. There is a small transfer of mass from the envelope to the supercritical core during the post-PMF epoch; by the time $\Delta t_{6}$ the core mass has increased by $8 \%$ to $5.80 M_{\odot}$. Figure $6 b$ displays $B_{z, \text { eq }}$ normalized to $B_{z, \text { eq,c0 }}$, Figure $6 c$ shows the ratio $\sigma_{\mathrm{n}} / \sigma_{\mathrm{n}, \mathrm{c} 0}$, and Figure $6 d$ depicts $B_{r, Z}$, the $r$ component of the magnetic field at the upper surface of

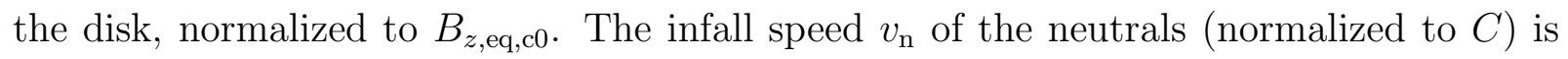
shown in Figure 6e, the ion-neutral drift speed $v_{\mathrm{D}}$ (also normalized to $C$ ) is displayed in Figure $6 f$, and the mass accretion rate $\dot{M}$ in $M_{\odot} \mathrm{Myr}^{-1}\left(=0.65 C^{3} / G\right.$ for the temperature and gas composition assumed in this model) is exhibited in Figure $6 g$. The ratio $\tau_{\text {ni }} / \tau_{\text {gr }}$ is plotted in Figure $6 h$, and the ratio $Z / r$ is shown in Figure $6 i$.

Taken together, Figures $6 b-6 i$ show the effect of ambipolar diffusion and the outward progression of the HMD (and the resulting hydromagnetic shock) on the evolution of the core. For $\Delta t<\Delta t_{2}$, ambipolar diffusion has not yet been revitalized, and mass and magnetic flux are continually advected inward. During this period, $\sigma_{\mathrm{n}}$ and $B_{z \text {,eq }}$ in the core decrease with increasing $\Delta t . B_{r, Z}$ increases as field lines are bent inward while being 
carried along with the neutrals. For $\Delta t_{j}>\Delta t_{2}$, ambipolar diffusion has become rapid enough to halt the advection in the innermost cells. Subsequently the front of piled-up magnetic flux expands outward to larger radii, dramatically enhancing the value of $B_{z \text {,eq }}$ in its wake. Bending of the interior field lines is slowed and temporarily arrested in the region of rapid ambipolar diffusion behind the HMD for $\Delta t_{2} \lesssim \Delta t \lesssim \Delta t_{5}$ (the behavior for $\Delta t>\Delta t_{5}$ is considered below). Further out from the center, the magnitude of the local gravitational field strength decreases while the degree of ionization increases, resulting in less efficient ambipolar diffusion and more effective collisional coupling between the ion and neutral fluids. At times $\Delta t \gtrsim \Delta t_{4}$ the HMD is propagating out into the regions of the core $\left(r / R_{0} \gtrsim 10^{-4}\right)$ where $\tau_{\text {ni }} / \tau_{\text {gr }} \lesssim 1$ (see Fig. $6 h$ ). At these radii both the collisional coupling of the ion and neutral fluids and the enhanced magnetic field strength behind the HMD are large enough to affect the inflow of the neutrals, and a shock forms. Our numerical calculation thus confirms the evolutionary sequence originally outlined in Li \& McKee (1996). A local maximum in $\sigma_{\mathrm{n}}$ occurs because the neutrals are "hung up" (i.e., decelerated; see Fig. 6e) in the region of stronger magnetic field strength, and their infall is interrupted as they are forced to diffuse through the stationary, or slowly expanding, ions and field lines contained in the HMD $\left(v_{\mathrm{D}} \approx\left|v_{\mathrm{n}}\right|\right.$ and $\left|v_{\mathrm{i}}\right| \ll\left|v_{\mathrm{n}}\right|$ behind the HMD; see Fig. $\left.6 f\right)$. After the neutrals diffuse through the shock, they resume dynamical collapse toward the origin: at time $\Delta t_{6},\left|a_{\mathrm{n}}\right| \gtrsim 0.90\left|g_{r}\right|$ for $r \lesssim 4 \times 10^{-5} R_{0}=35.5 \mathrm{AU}, \sigma_{\mathrm{n}} \propto r^{-1 / 2}$, and $v_{\mathrm{n}} \propto r^{-1 / 2}$ (which is the characteristic behavior of free-fall collapse induced by a central point mass, also found in self-similar models of gravitational collapse in isothermal disks; Li \& Shu 1997; CCK97). At that time $\left|v_{\mathrm{n}}\right|$ is a fraction $\sim 0.94$ of the free-fall speed $(\simeq 76.46 C)$ at $r=r_{\text {inner}}$. Depletion of matter, caused by the interruption in the infall of the neutrals at the shock front, is the reason for the local minimum in $\sigma_{\mathrm{n}}$ that occurs between the shock front and the free-fall region. This causes the accretion rate behind the shock to decrease for $\Delta t \gtrsim \Delta t_{4}$. Hence ambipolar diffusion, through the action of the hydromagnetic shock, has increased the magnetic field strength in the collapsing core for $r \gtrsim 10^{-4} R_{0}(\simeq 88.7 \mathrm{AU})$ and reduced the accretion rate onto the central protostar - exactly the opposite of what one would have expected a priori.

For comparison we also plot in Figures $6 b, 6 c$, and $6 d$ (dashed curves), for the model in which flux freezing into the neutrals was assumed to hold throughout the post-PMF epoch, the profiles of $\sigma_{\mathrm{n}}, B_{z \text {,eq }}$, and $B_{r, Z}$, respectively, at the time $\Delta t_{6}$ the mass of the central protostar reached $M_{\text {cent }}=1 M_{\odot}$. (For this model, $\Delta t_{6}=1.23 \times 10^{5}$ yr.) In the absence of ambipolar diffusion, the magnetic flux in the core is continually advected into the central sink, and $B_{z \text {,eq }}$ is greatly reduced from the value that characterizes the core of the typical ambipolar-diffusion model (see Fig. 6b); $B_{r, Z}$, on the other hand, is much larger in the core of the frozen-flux model (see Fig. 6c) because the field lines continue to be 
bent inward as mass and flux are advected into the central sink. Examination of Figure $6 c$ reveals that the column density of the ambipolar-diffusion model is significantly larger than that of the frozen-flux model for $r \gtrsim 1.6 \times 10^{-4} R_{0}=142 \mathrm{AU}$, i.e., the region just behind the shock front. For $r \lesssim 142$ AU the opposite occurs: $\sigma_{\mathrm{n}}$ in the model that includes ambipolar diffusion is much smaller than in the frozen-flux case. This is because more of the core mass remains further away from the center in the ambipolar-diffusion model, being trapped at larger radii by the pressure of the enhanced magnetic field in the wake of the HMD. Hence, ambipolar diffusion, by giving rise to a hydromagnetic shock, has significantly altered the evolution of the column density profile in the core. Moreover, a hydromagnetic shock does not occur in the core of the frozen-flux model. This is contrary to the results of the self-similar model of Li \& Shu (1997). This discrepancy arises because the initial conditions assumed by Li \& Shu correspond to a stationary configuration rather than to the dynamical one indicated by the numerical simulations of the pre-PMF phase of collapse (see $\S 3.2)$. In fact, as discussed in CCK97, shocks also do not form in the absence of ambipolar diffusion in self-similar models that do not use the initial singular-isothermal-disk setup of Li \& Shu. Finally, we note that, for $r \gtrsim R_{\text {crit }}(\Delta t)$, the magnetic field is the same in both models, reflecting the fact that ambipolar diffusion is so ineffective in the massive, subcritical envelope that the field there can be considered to be frozen into the neutrals at all times.

Figure $6 b$ shows that, by the time $\Delta t_{6}, B_{z \text {,eq }}$ has been significantly reduced for $r \lesssim 2.5 \times 10^{-4} R_{0}=222 \mathrm{AU}$. This is due to the fact that the drift speed $v_{\mathrm{D}}(r)$ exceeded the critical value $v_{\mathrm{D}, \text { crit }} \approx 12 \mathrm{~km} \mathrm{~s}^{-1}$ in this region of the core for $\Delta t_{5}<\Delta t<\Delta t_{6}$. Therefore, during this period, the neutral-ion collisional force scaled quadratically with $v_{\mathrm{D}}$ rather than linearly (compare eqs. [10] and [14]), so the frictional force between the ions and the neutrals was significantly increased in this part of the core. This had the effect of refreezing the magnetic flux into the neutrals in the innermost computational cells, resulting in the field being dragged by the collapsing neutrals into the central sink and therefore decreasing in strength in these cells. However, further out $\left(r>2.5 \times 10^{-4} R_{0}\right)$, where $v_{\mathrm{D}}(r)<v_{\mathrm{D}, \text { crit }}$, the bulk of the flux front remains largely unaffected by the collapse of its "floor." By $\Delta t_{6}$, though, $v_{\mathrm{D}}$ has fallen below $v_{\mathrm{D}, \text { crit }}$ (see Fig. $6 \mathrm{f}$ ) even in the innermost cells of the computational mesh. Bending of the field lines resumes in this region of the core at that time, with a subsequent increase in $B_{r, Z}$ (see Fig. $6 d$ ).

We note that beyond the core radius [i.e., for $r \geq R_{\text {crit }}(\Delta t) \simeq 2.52 \times 10^{-2} R_{0}=0.11 \mathrm{pc}$ ] $\left|v_{\mathrm{n}}\right| \ll C$ (see Fig. 6e), reflecting effective support of the massive, subcritical envelope by magnetic forces. Ambipolar diffusion in the envelope is so ineffective (resulting from the much greater relative abundance of ions, due to ionization by the external UV radiation field) that $v_{\mathrm{D}} \ll C$ (see Fig. $6 f$ ), and the magnetic field there can be considered to be 
frozen into the neutrals; furthermore, $B_{r, Z} \ll B_{z \text {,eq }}$ in the envelope (see Figs. $6 b$ and $6 d$ ), indicating that the field lines there remain essentially straight and parallel. Effective separation of the supercritical core and the envelope continues for $\Delta t \geq 0$, as can be seen by the dramatic drop (by more than four orders of magnitude) in $\dot{M}$ for $r \gtrsim R_{\text {crit }}\left(\Delta t_{j}\right.$ ) (see Fig. $6 g$ ). As noted in $\S 1$, Safier et al. (1997) modeled the evolution of a cloud's envelope during the post-PMF epoch. Equation (104) of Safier et al. gives their predicted accretion rate as a function of the initial cloud mass, radius, and the ratio of the initial mean and central density. Evaluating their expression for the parameters used in our typical model $\left(M_{\mathrm{d}}=98.3 M_{\odot}, R_{0}=4.29 \mathrm{pc}\right.$, and $\left.\left\langle n_{\mathrm{n}}\right\rangle_{0} / n_{\mathrm{n}, \mathrm{c} 0}=0.144\right)$ yields a spatially uniform envelope accretion rate $\dot{M}=3.7 M_{\odot} \mathrm{Myr}^{-1}$, which is close to our calculated accretion rate at the core boundary (see Fig. $6 g$ ). However, for $r \gtrsim 0.26 R_{0} \simeq 0.11$ pc their predicted value of $\dot{M}$ is several orders of magnitude higher than the one we have obtained in the envelope of our typical model. The difference can be attributed to the fact that Safier et al. chose to study model clouds for which ionization by the external interstellar UV radiation field was unimportant, resulting in a lower degree of ionization and a correspondingly higher ambipolar diffusion rate in the cloud envelope.

Finally, the effect that the tidal gravitational field of the central protostar (see eq. [9]) has on the vertical structure of the cloud can be seen in Figure $6 i$. For times $\Delta t>0$ the inner region of the core near the central mass is significantly compressed by the tidal force, and $Z / r$ becomes $\ll 1$. Further compression will be provided by the squeezing effect of the radial magnetic field component (see eq. [9]), particularly at late times in the vicinity of $r_{\text {inner }}$. All in all, the disk remains geometrically thin at all radii $r>r_{\text {inner }}$ for the entire duration of the simulation.

\section{Discussion}

\subsection{Observational Comparisons and Predictions}

We may compare our result for the protostellar accretion rate during the PMF epoch with observations of star-forming cores. As shown in $\S 3.3$, the accretion rate rises rapidly early on to $\dot{M}_{\text {cent }} \simeq 9.4 M_{\odot} \mathrm{Myr}^{-1}$ for $\Delta t \lesssim 10^{3}$ yr (see Fig. $2 b$ ) and stays at this value up to the formation of the hydromagnetic shock. For $\Delta t z 4 \times 10^{3} \mathrm{yr}$ the shock is able to decelerate the infalling matter, and the accretion rate decreases to $\dot{M}_{\text {cent }} \simeq 5.6 M_{\odot} \mathrm{Myr}^{-1}$ by $\Delta t \simeq 1.5 \times 10^{5}$ yr (the time when $M_{\text {cent }}=1 M_{\odot}$ ). Therefore $\dot{M}_{\text {cent }}$ decreases with increasing central mass (see Fig. 2c). This is consistent with estimates of ages and accretion rates $\left(\propto t_{\text {age }}^{-1}\right)$ for young stellar objects, as deduced from evolutionary diagrams inferred from observations of Class 0 and Class I objects (e.g., Saraceno et al. 1996). In particular, 
the lifetimes of Class 0 objects were estimated in this way to be an order of magnitude shorter than those of Class I objects, providing evidence for a decrease in the protostellar accretion rate as an object evolves from a Class 0 source to a Class I source (e.g., André 1995; Ward-Thompson 1996). Another argument for a time-dependent accretion rate was given by Bontemps et al. (1996), who analyzed the observed CO momentum flux of several young stellar objects and found a noticeable decline in the CO flux with decreasing circumstellar envelope mass. They suggested that this is indicative of a decrease in the protostellar accretion rate (which they assumed to be proportional to the mass outflow rate) as an object evolves from Class 0 to Class I.

It is also of interest to note the ${ }^{13} \mathrm{CO}(J=1-0)$ observations of infalling disks for the protostellar candidates HL Tauri (Hayashi, Ohashi, \& Miyama 1993) and L1551-IRS5 (Ohashi et al. 1996). HL Tauri has a mass $\sim 0.6 M_{\odot}$ and a surrounding disk with radius $\sim 1400 \mathrm{AU}$ and mass $\sim 0.03 M_{\odot}$. From the observed kinematics Hayashi et al. derive an accretion rate $\sim 9 M_{\odot} \mathrm{Myr}^{-1}$ at $r \sim 700 \mathrm{AU}$. The embedded protostar L1551-IRS5 has a mass $\sim 0.5 M_{\odot}$ and is surrounded by a disk with radius $\sim 700 \mathrm{AU}$ and mass in the range $3.9 \times 10^{-2}-8.1 \times 10^{-2} M_{\odot}$. Ohashi et al. deduce an accretion rate in the range $13-26 M_{\odot} \mathrm{Myr}^{-1}$ at $r \sim 600$ AU. These values are comparable to our model results for $\Delta t_{5} \lesssim \Delta t \lesssim \Delta t_{6}$ (corresponding to $0.2 M_{\odot} \lesssim M_{\text {cent }} \lesssim 1 M_{\odot}$; see Fig. $6 a$ ). During this period, $6 M_{\odot} \mathrm{Myr}^{-1} \lesssim \dot{M} \lesssim 9 M_{\odot} \mathrm{Myr}^{-1}$ for $r \gtrsim 500 \mathrm{AU}$ (see Fig. 6g). [Note, however, that the temperatures of HL Tauri and L1551-IRS5 are in the range $15-50 \mathrm{~K}$, which is greater than our assumed value of $10 \mathrm{~K}$ and should lead to higher values of $\dot{M}$ and $M_{\text {cent }}(t)$; e.g., Shu et al. 1987. For a discussion of how quantities scale with temperature in our models, see Basu \& Mouschovias 1994.] In Figure 7 we show the mass $\left(M-M_{\text {cent }}\right)$ of the gas surrounding the central point mass in our typical model as a function of $r / R_{0}$ for the same seven times $\Delta t_{j}$ as in Figure 6. (Taken together, Figs. $6 a$ and 7 may be taken to represent the evolution of a protostar from a Class 0 to a Class I object.) For times $\gtrsim \Delta t_{5}$, the surrounding disk mass spans the range $0.01-0.1 M_{\odot}$ for $r \gtrsim 500 \mathrm{AU}$, which agrees with the ${ }^{13} \mathrm{CO}$ disk masses of HL Tauri and L1551-IRS5 cited above. Finally, we note that the age of the oldest part of the molecular outflow from L1551-IRS5 is estimated to be $\sim 10^{5} \mathrm{yr}$ (Bachiller, Tafalla, \& Cernicharo 1994). This age is consistent with the time $\Delta t$ needed for the central mass in our typical model to become $\gtrsim 0.3 M_{\odot}$ (see Fig. $2 e$ ).

Another observational consequence of our model is the magnetic field structure in the core after PMF. Figures $6 b$ and $6 d$ show that $B_{r, Z} \approx B_{z \text {,eq }}$ inside the core for the radius range $2 \times 10^{-4} \lesssim r / R_{0} \lesssim 4 \times 10^{-3}$. Hence, there is significant curvature of field lines (though, as discussed in $\S 3.3$, there is less bending than there would be if the field had remained frozen into the neutrals), with bending $\operatorname{angles} \theta_{B} \approx \arctan \left(B_{r, Z} / B_{z \text {,eq }}\right)$ in the 
range $20^{\circ}-50^{\circ}$ for $180 \mathrm{AU} \lesssim r \lesssim 3.5 \times 10^{3} \mathrm{AU}$. 10 This type of field geometry may be described roughly as having an hourglass shape. In fact, sub-mm polarimetry of the cores of W3 IRS5 (Greaves, Murray, \& Holland 1994), Mon R2 (Greaves, Holland, \& Murray 1995), and OMC-1 (Schleuning 1998) find field geometries suggestive of an hourglass shape on sub-parsec scales. In contrast, field lines remain essentially straight and parallel in the magnetically supported envelope $(r>0.1 \mathrm{pc})$, even after PMF. This agrees with polarimetric observations of molecular clouds that indicate well-ordered fields on these scales (e.g., Hildebrand, Dragovan, \& Novak 1984; Hildebrand 1989, 1996; Novak et al. 1989; Kane et al. 1993; Hildebrand et al. 1995).

A unique prediction of our model is the large ion-neutral drift speed that occurs during the post-PMF epoch. As shown in Figure $6 f$, effective ambipolar diffusion following PMF yields $v_{\mathrm{D}} \approx\left|v_{\mathrm{n}}\right| \gg C$ in the inner regions of the core. In our typical model we find $v_{\mathrm{D}} \gtrsim 1 \mathrm{~km} \mathrm{~s}^{-1}$ for $\Delta t \gtrsim 2 \times 10^{4}$ yr on scales $r \lesssim 2 \times 10^{-4} R_{0} \simeq 180$ AU. Large drift speeds between neutrals and ions (such as $\mathrm{HCO}^{+}, \mathrm{HCN}^{+}, \mathrm{DCO}^{+}$, to name but a few) on these scales are therefore expected in our model. Detection of such drift speeds (through high-resolution observations of $\mathrm{HCN}$ or $\mathrm{HCO}^{+}$, say) could be used to observationally confirm our model results and to distinguish them from those of nonmagnetic collapse calculations (e.g., Shu 1977, Hunter 1977, Foster \& Chevalier 1993) or of magnetic collapse models that do not account for the effect of ambipolar diffusion (e.g., Tomisaka 1996; Li \& Shu 1997).

\subsection{Features of the Hydromagnetic Shock}

The properties of hydromagnetic shocks in partially ionized gases have been developed extensively by many other authors (e.g., Mullan 1971; Draine 1980; Chernoff 1987; Roberge \& Draine 1990; Draine \& McKee 1993; Smith \& Mac Low 1997). Because the ion Alfvén speed $v_{\mathrm{A}, \mathrm{i}}=B_{z \text {,eq }} /\left(4 \pi m_{\mathrm{i}} n_{\mathrm{i}}\right)^{1 / 2}=\left(m_{\mathrm{n}} / m_{\mathrm{i}}\right)^{1 / 2}\left(n_{\mathrm{n}} / n_{\mathrm{i}}\right)^{1 / 2} v_{\mathrm{A}}$ is much larger than $v_{\mathrm{A}},\left|v_{\mathrm{n}}\right|$, and $\left|v_{\mathrm{i}}\right|$ in our model, we expect the outward-propagating shock that develops after PMF to have a magnetic precursor. This is indeed what we find in our simulation: the jump in the ion speed $v_{\mathrm{i}}$ and the magnetic field strength $B_{z, \text { eq }}$ typically occurs at a distance of 1 to 3 computational mesh spacings further away from the symmetry axis than the jump in the neutral infall speed $v_{\mathrm{n}}$ and the column density $\sigma_{\mathrm{n}}$. The displacement between the locations

\footnotetext{
${ }^{10}$ In agreement with the results of CM94 and CM95, we find that the magnetic tension force is generally not negligible in comparison with the magnetic pressure force at any radius (in either the core or the envelope), both before and after PMF.
} 
of the head of the disturbance in the neutral fluid and in the plasma and magnetic field decreases at later times. Examination of Figures $5 b, 5 c$, and $6 e$ reveals that, in the reference frame of the shock, the preshock infall speeds are supersonic, and the postshock speeds are also supersonic or just slightly subsonic. Similarly, the ion infall speeds (in the frame of the shock) are much less than the ion Alfvén speed. Therefore the shock we observe in our model is probably best classified as being C-type. 四 Making the approximation that in the vicinity of the shock the predominant magnetic stress is that due to the magnetic pressure gradient, the ion force equation becomes

$$
\frac{\sigma_{\mathrm{n}}}{\tau_{\mathrm{ni}}} v_{\mathrm{D}}=-\frac{Z}{4 \pi} \frac{\partial B_{z, \mathrm{eq}}^{2}}{\partial r}
$$

(see eqs. [28c] and [51] in CM93, which contain additional terms, involving in particular the magnetic tension force, that could be used to refine the following simple estimate). This yields an approximate shock width

$$
\begin{aligned}
& \Delta_{\mathrm{shk}} \approx \frac{B_{z, \mathrm{eq}, \mathrm{u}}^{2}}{4 \pi \sigma_{\mathrm{n}}} \frac{\tau_{\mathrm{ni}} Z}{v_{\mathrm{D}}} {\left[\left(\frac{B_{z, \mathrm{eq}, \mathrm{d}}}{B_{z, \mathrm{eq}, \mathrm{u}}}\right)^{2}-1\right]=C \tau_{\mathrm{ni}} \frac{\left(v_{\mathrm{A}, \mathrm{u}} / C\right)^{2}}{2\left(v_{\mathrm{D}} / C\right)}\left[\left(\frac{B_{z, \mathrm{eq}, \mathrm{d}}}{B_{z, \mathrm{eq}, \mathrm{u}}}\right)^{2}-1\right] } \\
&=7.8 \times 10^{13} \frac{(T / 10 \mathrm{~K})^{1 / 2}\left(v_{\mathrm{A}, \mathrm{u}} / C\right)^{2}}{\left(m_{\mathrm{n}} / 2.33 \text { a.m.u. }\right)^{1 / 2}\left(n_{\mathrm{i}} / 0.1 \mathrm{~cm}^{-3}\right)\left(v_{\mathrm{D}} / C\right)}\left[1+0.067 \frac{\left(m_{\mathrm{H}_{2}} / 2 \text { a.m.u. }\right)}{\left(m_{\mathrm{i}} / 30 \text { a.m.u. }\right)}\right] \\
& \times\left[\left(\frac{B_{z, \mathrm{eq}, \mathrm{d}}}{B_{z, \text { eq }, \mathrm{u}}}\right)^{2}-1\right] \mathrm{cm},
\end{aligned}
$$

where $B_{z, \text { eq,u }}$ and $B_{z, \text { eq,d }}$ are the values of $B_{z, \text { eq }}$ upstream and downstream of the shock, and $v_{\mathrm{A}, \mathrm{u}}$ is the upstream Alfvén speed. In deriving the last equality of equation (17a) we have used the relation $\sigma_{\mathrm{n}}=2 \rho_{\mathrm{n}} Z$; equation (1) and the relation $C=\left(k_{\mathrm{B}} T / m_{\mathrm{n}}\right)^{1 / 2}$ have been used in deriving equation (17b). At the time $\Delta t_{6}$ the shock front is located at $r \simeq 3.9 \times 10^{-3} R_{0}=5.2 \times 10^{16} \mathrm{~cm}$, and, in the vicinity of the front, $n_{\mathrm{i}} \simeq 10^{-2} \mathrm{~cm}^{-3}$, $v_{\mathrm{A}, \mathrm{u}} \simeq C, B_{z, \mathrm{eq}, \mathrm{d}} / B_{z, \mathrm{eq}, \mathrm{u}} \simeq 4.6$ (see Fig. $6 b$ ), and $v_{\mathrm{D}} \simeq 0.4 C$ (see Fig. $6 f$ ). For these values our rough estimate for the shock width given by equation (17b) yields $\Delta_{\text {shk }} \simeq 4.3 \times 10^{16} \mathrm{~cm}$. Examination of Figure $6 e$ at the time $\Delta t_{6}$ reveals that the shock has an actual width $\Delta_{\text {shk }} \simeq 2.1 \times 10^{-3} R_{0}=2.8 \times 10^{16} \mathrm{~cm}$. (Thus $\Delta_{\text {shk }} / r_{\text {shk }} \approx 0.5$ at that time, so the thin-shock approximation that underlies the estimate [17] is marginally satisfied.)

\footnotetext{
${ }^{11} \mathrm{~A}$ C-type shock is characterized by neutral velocities that (in the shock frame) remain supersonic throughout. Hence the shock in our simulation cannot be strictly of this type when the downstream neutral speed is subsonic. In that case a viscous (J-type) subshock may form (Draine \& McKee 1993), although, as noted by Li \& McKee (1996), turbulent diffusivity behind a real shock could plausibly keep the postshock flow supersonic and thereby obviate the need for such a subshock.
} 
As noted in $\S 1$, Li \& McKee (1996) proposed that a hydromagnetic shock would form in a collapsing core as a result of the decoupling of flux from the ion and neutral fluids because of Ohmic dissipation (a process that becomes important in regions of density $n_{\mathrm{n}} \gg 10^{11} \mathrm{~cm}^{-3}$; see $\S 2.1$. They argued that the accumulating flux diffuses outward to regions of lower density, where improved coupling with the matter causes it to present an obstacle to the infalling neutral gas - thereby giving rise to a hydromagnetic shock. While our numerical results have confirmed Li \& McKee's basic shock-formation scenario, they have revealed that ambipolar diffusion, which mediates the shock, can also, following PMF, halt the inward advection of magnetic flux on scales $(r \gtrsim 5 \mathrm{AU})$ where Ohmic dissipation is not important. In other words, our results have shown that, during the post-PMF epoch, the field-matter decoupling that drives the hydromagnetic shock is due to ambipolar diffusion alone and does not depend on the effect of Ohmic dissipation at $r<r_{\text {inner }}$.

Because of the similarity in the basic shock-formation mechanism, it is of interest to compare our detailed simulation results with the predictions of the (simplified and analytic) shock model of Li \& McKee (1996). From their requirement that the magnetic pressure of the shock balance the ram pressure of the neutrals, which were assumed to be freely-falling into the shock, Li \& McKee derived relations for the shocked magnetic field strength and the shock location (see their eqs. [7] and [8]) in terms of the accretion rate $\dot{M}$, the flux-to-mass ratio in units of the critical value for collapse (dubbed $\epsilon$ in their paper), a parameter related to the logarithmic gradient of the magnetic field (dubbed $\chi$ ), the ratio $Z / r$ of the local gas scale height and the radius (dubbed $h$ ), and the protostellar mass (denoted by $m_{*}$ ). At the time $\Delta t_{6}$ we have at the location of our shock $\dot{M} \approx 9 M_{\odot} \mathrm{Myr}^{-1}$, $\epsilon \approx 0.9, \chi \approx 2, h \approx 0.3$, and $m_{*}=M_{\text {cent }}\left(\Delta t_{6}\right) \approx 1 M_{\odot}$. Inserting these values into their equations (7) and (8) yields a shocked magnetic field strength $\sim 630 \mu \mathrm{G}$ and a shock radius $\sim 2.1 \times 10^{3} \mathrm{AU}$; by comparison, in our model the shocked magnetic field strength at that time is $B_{z, \text { eq,d }} \approx 330 \mu \mathrm{G}$ and the shock radius is $r_{\text {shk }} \approx 3.5 \times 10^{3}$ AU (see Figs. $6 b$ and $6 e$ ). The main reason why the analytic expression overestimates the numerically calculated magnetic field strength is that, contrary to the assumption of Li \& McKee, the preshock neutrals are not in free fall but, rather, are strongly decelerated by magnetic forces (in our simulation we find that the preshock acceleration of the neutrals is reduced to $\simeq 0.25 g_{r}$ ). The corresponding reduction in the preshock ram pressure leads to a lower value of the postshock field amplitude, with a further reduction in the calculated field strength brought about by the contribution of magnetic tension (ignored in the analytic estimate) to the total magnetic force. Since the analytic estimate of $r_{\text {shk }}$ is based on relating the postshock field strength to the total magnetic flux inside the shock, the overestimate of the field strength naturally results in an underestimate of the shock radius. Despite these discrepancies, Li \& McKee's analytic representation of the shock parameters provides a decent approximation 
to the results of our numerical calculation.

Comparison of the column density $\sigma_{\mathrm{n}}$ (see Fig. 6c) and neutral infall speed $v_{\mathrm{n}}$ (see Fig. $6 e$ ) behind the shock shows qualitative agreement with Figures $2 a$ and $2 d$ of Li \& McKee (1996), including the free-fall behavior near the central protostar. In the pre-shock region, however, our infall speeds are smaller than theirs because of their assumption of free fall upstream of the shock: Li \& McKee typically overestimate $v_{\mathrm{n}, \mathrm{u}}$ by a factor $\sim 2$. As a result, the Mach number of the shock relative to the upstream gas (and thus the shock strength) is greater in their model than in ours (see Fig. 5c) by a similar factor. We have not compared our results for the magnetic field structure behind the shock with those of Li \& McKee on account of the fact that their system of MHD equations was not closed (it did not include the magnetic induction equation), so that they were unable to calculate the magnetic field with any accuracy (see their Fig. 2b).

\subsection{Stability of the Core Against Magnetic Interchange}

Our simulation has revealed that rapid ambipolar diffusion occurs behind the outwardpropagating HMD. The effect that this has on the mass in the flux tubes downstream of the HMD can be seen in Figure $8 a$, which shows the local mass-to-flux ratio $d M / d \Phi_{B}=\sigma_{\mathrm{n}} / B_{z \text {,eq }}$ (normalized to the critical value for collapse) as a function of $r / R_{0}$ for the same seven times $\Delta t_{j}$ as in Figure 6. For $\Delta t>\Delta t_{1}$ a local minimum in $\sigma_{\mathrm{n}} / B_{z \text {,eq }}$ appears after the passage of the HMD. Hence, there is a region behind the HMD for which $d\left(\sigma_{\mathrm{n}} / B_{z, \text { eq }}\right) / d r>0$. This is a necessary condition for the onset of an interchange instability (e.g., Spruit \& Taam 1990; Lubow \& Spruit 1995; Spruit, Stehle, \& Papaloizou 1995). Li \& McKee (1996) speculated that such a situation could arise in the wake of a hydromagnetic shock in a collapsing core and suggested that it would act as source of turbulence in the postshock region of the inflow. 12

Blaes \& Balbus (1994) found that the magnetic shearing instability in differentially rotating disks could be stabilized if the disk is weakly ionized. This will also be the case for the interchange instability in a weakly ionized disk: instability is possible only if the growth rate $\gamma_{\text {II }}$ and the neutral-ion collision time $\tau_{\text {ni }}$ satisfy the condition $\gamma_{\text {II }} \tau_{\text {ni }}<1$. This condition reflects the fact that there has to be sufficient collisional coupling between the ion

\footnotetext{
${ }^{12} \mathrm{Li} \&$ McKee (1996) also noted that the shock may be unstable to the Wardle instability, which involves ions collecting in magnetic field "valleys" (Wardle 1990). However, the shock will be immune to this instability if the ion density is determined by the local chemical reaction balance (as assumed in our calculation) rather than by the divergence of the ion mass flux.
} 
and neutral fluids for a magnetic interchange instability to grow in the neutrals; otherwise the instability is damped. Calculation of $\gamma_{\mathrm{II}}$ in our model is hampered by the fact that previous studies of interchange instability have been carried out only for disks that are in hydrostatic equilibrium, with exact balance between magnetic and gravitational forces. We can apply the results of these studies to our model only if the region behind the shock where $d\left(\sigma_{\mathrm{n}} / B_{z, \text { eq }}\right) / d r>0$ is effectively in quasi equilibrium, with approximate balance between gravitational and magnetic forces, and with infall speeds $\left|v_{\mathrm{n}}\right| \ll\left(r\left|g_{r}\right|\right)^{1 / 2}$ ( $\simeq$ the free-fall speed). In our model, the magnitude of the acceleration of the neutrals in this region of the core does not become $\lesssim 0.1\left|g_{r}\right|$ until times $\sim \Delta t_{6}$; hence, approximate equilibrium between magnetic and gravitational forces is valid only at these later times. Spruit \& Taam (1990) found that the growth rate for the most unstable linear interchange modes is

$$
\gamma_{\mathrm{II}}=\left(\frac{B_{z, \mathrm{eq}} B_{r, Z}}{2 \pi \sigma_{\mathrm{n}}} \frac{d}{d r} \ln \frac{\sigma_{\mathrm{n}}}{B_{z, \mathrm{eq}}}\right)^{1 / 2}
$$

The product $\gamma_{\mathrm{II}} \tau_{\mathrm{ni}}$ for the region of the core susceptible to interchange instability is shown in Figure $8 b$ as a function of $r / R_{0}$ at the time $\Delta t_{6}$. We also plot (Fig. $8 c$ ) the product $\gamma_{\text {II }} \tau_{\text {kin }}$, where $\tau_{\text {kin }} \equiv r /\left|v_{\mathrm{n}}\right|$ is the kinematical timescale, as a function of $r / R_{0}$ for the same region of the core and time. If this product is $<1$, the unstable modes will be "swept up" by the infalling gas before they have time to grow. It is evident from these figures that $\gamma_{\mathrm{II}} \tau_{\mathrm{ni}}<1$ and $\gamma_{\mathrm{II}} \tau_{\text {kin }}>1$ for the region of the core susceptible to magnetic interchange. This means that there is sufficient collisional coupling between the ions and neutrals, and that the instability will grow before being swept along with the neutrals. Hence, this region of the core may be interchange unstable. An instability of this type would enhance the tansfer of gas with a high mass-to-flux ratio to the center (e.g., Spruit \& Taam 1990), and, as noted by Li \& McKee (1996), might also lead to the development of turbulence that could increase the field diffusivity in the postshock gas. However, the onset and development of this instability can only be studied by means of a fully $3-\mathrm{D}$ simulation.

\subsection{Implications to the Magnetic Flux Problem}

The magnetic flux problem in star formation has to do with the fact that the magnetic flux of a $1 M_{\odot}$ blob of matter in the diffuse interstellar medium is typically several orders of magnitude greater than the flux of a $1 M_{\odot}$ protostar. Such a blob of matter would therefore have to get rid of most of its flux before becoming a star. Ambipolar diffusion has long been suggested as a means by which the magnetic flux problem could be resolved (e.g., Mestel \& Spitzer 1956; Mouschovias 1978; Paleologou \& Mouschovias 1983; Nakano 1984; Mouschovias, Paleologou, \& Fiedler 1985). In general, these earlier studies focused primarily 
on the role of ambipolar diffusion and the magnetic flux problem for the pre-PMF phase of protostellar evolution. [15 While ambipolar diffusion does indeed reduce the flux-to-mass ratio during that phase, the flux contained within a $1 M_{\odot}$ region of a molecular cloud core is still much larger at the time of PMF than typical protostellar fluxes. Specifically, CM94 and CM95 found that the central $1 M_{\odot}$ flux tube within their cores had a total magnetic flux $\sim 10^{30} \mathrm{Mx}$ (consistent with our results in $\S 3.2$ ) during the pre-PMF dynamical collapse phase of the typical model. This value represents a reduction by a factor $\sim 5.6$ of the flux associated with that mass before the onset of ambipolar diffusion. Nevertheless, it greatly exceeds the plausible upper limit $\left(\sim 6 \times 10^{26} \mathrm{Mx}\right)$ on the flux of a solar-mass protostar (estimated assuming an average surface field of $10 \mathrm{kG}$ and a stellar radius of $10^{11} \mathrm{~cm}$; see Li \& McKee 1996).

We have shown in this paper that the rate of ambipolar diffusion is strongly increased during the post-PMF epoch of star formation. It is therefore of interest to examine the implications of our simulation results to the magnetic flux problem. As discussed in $\S 2.2$, in our calculations we only consider the core regions at radii $r \gtrsim r_{\text {inner }}(\simeq 7.3 \mathrm{AU}$ for our typical model), where ambipolar diffusion is the dominant mechanism of flux loss. Initially, the magnetic flux contained within $r_{\text {inner }}$ is $\Phi_{B, \text { cent }}(\Delta t=0)=6.7 \times 10^{26} \mathrm{Mx}$. As shown in Figure $3 a$, the central flux increases before the onset of rapid ambipolar diffusion. This continues to the time $\Delta t \approx 10^{3} \mathrm{yr}$. For $\Delta t>10^{3} \mathrm{yr}$, ambipolar diffusion prevents further advection of flux from $r>r_{\text {inner }}$ into the central sink, and $\Phi_{B \text {,cent }}$ changes very little after this time. By the time $\Delta t \approx 10^{5} \mathrm{yr}$, when $M_{\text {cent }} \approx 1 M_{\odot}, \Phi_{B \text {, cent }} \approx 5 \times 10^{27} \mathrm{Mx}$. This represents a decrease of over two orders of magnitude relative to the flux associated with this mass at the time of PMF. While this value is still about an order of magnitude higher than our adopted upper limit on the protostelar flux, the discrepancy is now much lower than previous estimates of ambipolar diffusion have indicated. The important conclusion from our work is thus that ambipolar diffusion in contracting molecular cloud cores can in principle contribute significantly to the resolution of the magnetic flux problem by reducing the magnetic flux brought into a solar-mass protostar by a factor $z 10^{3}$. The new, and somewhat surprising, result is that most of this reduction occurs after PMF.

\footnotetext{
${ }^{13}$ On the basis of a consideration of the timescales for ambipolar diffusion and Ohmic dissipation at high densities, Nakano \& Umebayashi (1986b) suggested that significant flux loss could only take place (primarily by Ohmic dissipation, according to their estimates) during the dynamical phase of core collapse. Lizano \& Shu (1989) similarly concluded that the resolution of the protostellar magnetic flux problem must occur during the dynamical stage of core evolution: using the quasi-static approximation (valid for $n_{\mathrm{n}, \mathrm{c}} \lesssim$ a few $\times 10^{4} \mathrm{~cm}^{-3}$; Fiedler \& Mouschovias 1993; CM94; Basu \& Mouschovias 1994) to calculate the contraction of a slightly subcritical molecular cloud, they found that only a small amount of flux is lost by ambipolar diffusion from the central flux tubes before runaway collapse is initiated.
} 
The remainder of the protostellar magnetic flux could possibly be extracted from the infalling mass through Ohmic dissipation within $r_{\text {inner }}$, although refreezing of the magnetic field into the matter, brought about by collisional reionization at densities $n_{\mathrm{n}} \gtrsim 10^{14} \mathrm{~cm}^{-3}$ (e.g., Pneuman \& Mitchell 1965; Nakano \& Umebayashi 1986b; Li \& McKee 1996), as well as anomalous diffusivity (e.g., Norman \& Heyvaerts 1985) operating in the reionized gas, could complicate the issue. Another complicating factor is the strong likelihood that much of the mass and flux carried into the protostar pass through a rotationally supported, circumstellar accretion disk of size $\gg r_{\text {inner }}$ (e.g., Lubow, Papaloizou, \& Pringle 1994; Reyes-Ruiz \& Stepinski 1996; Li 1996). It is also conceivable that magnetic flux is brought to the vicinity of the protostar but excluded from its interior by turbulent diffusivity associated with convection. Since the region within $r_{\text {inner }}$ was excluded from our calculation, we do not pursue this topic any further in this paper.

\section{Summary}

We have simulated the formation and growth of a central (i.e., protostellar) point mass in a gravitationally collapsing core of a nonrotating magnetic cloud modeled as an isothermal and axisymmetric thin disk. Following up on the results of previous simulations, we concentrated in this study on the core evolution after point-mass formation (PMF), paying particular attention to the role of ambipolar diffusion during this phase. In view of our assumptions of gas isothermality and magnetic flux freezing into the ions, our results are applicable only on scales $r \gtrsim 5$ AU.

Just prior to the formation of a point mass, the model core is dynamically collapsing (though not freely falling), with infall speeds that are comparable to, or exceed, the isothermal speed of sound and the local fast-magnetosonic speed, and accelerations ranging from 0.25 to 0.5 of the local gravitational acceleration.

We have calculated the evolution of the central protostar up to the time that it has grown in mass to $1 M_{\odot}$. Ambipolar diffusion causes the evolution of our model core to differ significantly from that found in previous calculations of dynamically collapsing cores, which considered either nonmagnetic or magnetic but perfectly conducting clouds. In particular, we find that ambipolar diffusion in the weakly ionized gas surrounding the central protostar is "revitalized" by the increase in the strength of the gravitational field brought about by the formation and growth of the central point mass. (An alternative, but equivalent, explanation of this revitalization is that the magnetic tension force acting on the ions increases to the point where the ion-neutral drift speed becomes comparable to the neutral inflow speed.) The ambipolar diffusion becomes rapid enough to stop the inward advection 
of magnetic flux, which therefore begins to pile up in the inner regions of the collapsing core. The region of piled-up flux develops into a hydromagnetic disturbance that propagates outward and increases the local magnetic field amplitude away from the axis of symmetry. As the field increases and the disturbance reaches regions where the ion-neutral coupling becomes stronger, the hydromagnetic front evolves into a (C-type) shock: for the physical parameters assumed in our typical model, this occurs at radii $r \gtrsim 90 \mathrm{AU}$. The shock speed is supersonic (and super fast-magnetosonic) with respect to the infalling upstream gas, but for $r \gtrsim 140 \mathrm{AU}$ it becomes subsonic in the protostellar frame. The shock decelerates the neutrals and interrupts their infall, thereby decreasing the accretion rate onto the central protostar: we confirmed that the accretion rate obtained by accounting for the effect of ambipolar diffusion was less than that calculated in a model that evolved under the assumption of flux freezing into the neutrals. Far behind the shock (at radii $\sim 10-40 \mathrm{AU}$ in our typical model) the neutrals are reaccelerated to free-fall, and the column density and neutral infall speed scale as $r^{-1 / 2}$. The column density scales with the time $\Delta t$ since PMF as $\sigma_{\mathrm{n}} \propto(\Delta t)^{s} \Delta t$, with $s_{\Delta t}$ lying in the range $-0.76 \lesssim s_{\Delta t} \lesssim-0.55$ (whose upper bound is close to the value $s_{\Delta t}=-0.5$ obtained under the assumption of self-similarity). Our numerical results are in overall agreement with the simplified analytic model of Li \& McKee (1996), who first predicted the existence of the outward-propagating C-shock. We have found that, after PMF, this shock is driven primarily by ambipolar diffusion, which leads to field-matter decoupling on scales larger than those where Ohmic diffusivity effects are important. CCK97 derived a semianalytic similarity solution that incorporates ambipolar diffusion and captures the main features of the post-PMF core evolution (including the shock formation) found in our simulation.

For the typical model presented in this paper, the protostellar accretion rate increases from $\sim 5 M_{\odot} \mathrm{Myr}^{-1}$ just prior to protostar formation to a maximum $\sim 9.4 M_{\odot} \mathrm{Myr}^{-1}$ at $\Delta t \simeq 10^{3} \mathrm{yr}$ (although, because of the approximations involved in our calculation of the radial magnetic field component at the inner boundary, the actual maximum accretion rate might be higher). The accretion rate subsequently decreases, largely on account of the interruption of the infall by the hydromagnetic shock, and it is equal to $5.6 M_{\odot} \mathrm{Myr}^{-1}$ by the time $\left(1.5 \times 10^{5} \mathrm{yr}\right.$ after $\left.\mathrm{PMF}\right)$ the central mass has grown to $1 M_{\odot}$. For comparison, the "canonical" accretion rate $\left(\simeq C^{3} / G\right.$; Shu 1977) for this model is $1.5 M_{\odot} \mathrm{Myr}^{-1}$, although it has been recognized that this value would be larger in clouds where magnetic stresses supplement thermal pressure support. [For example, Li \& Shu (1997) estimated an increase by a factor $\sim\left(1+H_{0}\right)$, where $H_{0}(\sim 1)$ is the magnetically supported fractional overdensity in the stationary, pre-PMF cloud. Other magnetic models (e.g., Tomisaka 1996; Basu 1997) have also indicated a larger-than-canonical average accretion rate.] The decline in the accretion rate with time is consistent with the trend inferred from observations of Class 0 
and Class I sources. Furthermore, the accretion rate and the circumstellar mass at radii $\gtrsim 500 \mathrm{AU}$ in our typical model are comparable to those derived by millimeter observations of protostars with "infalling" disks, such as HL Tauri and L1551-IRS5. The magnetic field structure in our model is consistent with polarimetric observations of molecular clouds that reveal essentially uniform fields in cloud envelopes $(r \gtrsim 0.1 \mathrm{pc})$, and with sub-mm polarimetric surveys that indicate hourglass field structures within cloud cores.

Ambipolar diffusion is so effective after PMF that it leads to a reduction by more than two orders of magnitude in the flux threading the central $1 M_{\odot}$, compared with less than one order of magnitude reduction in the flux threading this mass between the start of the cloud contraction and the time of PMF. Altogether, ambipolar diffusion reduces the flux by more than three orders of magnitude and brings it to within an order of magnitude of the estimated upper limit on the flux of a $\sim 1 M_{\odot}$ protostar. Ambipolar diffusion in collapsing cloud cores could thus go a long way toward resolving the protostellar magnetic flux problem, although to fully address this issue one would need to consider Ohmic diffusivity on scales smaller than those included in the present calculation as well as the effects of rotation.

Large $\left(~ \gtrsim 1 \mathrm{~km} \mathrm{~s}^{-1}\right)$ ion-neutral drift speeds occur in our representative simulation on scales $r \lesssim 200 \mathrm{AU}$ for $\Delta t \gtrsim 2 \times 10^{4} \mathrm{yr}$, and are a unique prediction of our model. Observational detection of this effect could be used to distinguish our results from other (nonmagnetic and magnetic) collapse models that do not account for ambipolar diffusion. We have also confirmed that the postshock region is susceptible to the magnetic interchange instability, as first suggested by Li \& McKee (1996). This instability could only develop outside the region of large ion-neutral drift velocities, where it is not subject to quenching by ambipolar diffusion effects. Magnetic interchange would increase the rate of mass transfer to the center and could also lead to turbulence that might enhance the magnetic diffusivity. A numerical investigation of this instability does, however, require a $3-\mathrm{D}$ simulation.

The mass and magnetic flux distributions during the post-PMF epoch are directly relevant to the question of protostellar disk formation and the generation of disk outflows by magnetic ejection mechanisms (e.g., Königl \& Ruden 1993). However, in order to model the formation of centrifugally supported circumstellar disks, it is necessary to incorporate rotation and magnetic braking into the core-evolution calculations. This will be considered in a future publication.

This work was supported in part by NASA grants NAG 5-2266 and NAG 5-3687. We thank the referee, T. Hartquist, for several suggestions that helped improve the presentation of this paper. A discussion with R. Hildebrand on polarimetric observations 
of ordered magnetic fields in and around interstellar clouds is gratefully appreciated. We also acknowledge useful input from C. McKee, Z.-Y. Li, P. André, and F. Shu. GC would especially like to thank T. Mouschovias and S. Morton for early discussions on the nature and formulation of the problem presented in this paper.

\section{APPENDIX A}

\section{DESCRIPTION OF MODEL PARAMETERS}

We consider isothermal, magnetic interstellar molecular clouds consisting of neutral molecules $\left(\mathrm{H}_{2}\right.$ with a $20 \%$ He abundance), singly charged molecular and atomic ions (such as $\mathrm{HCO}^{+}, \mathrm{Mg}^{+}, \mathrm{Na}^{+}, \mathrm{Fe}^{+}, \mathrm{C}^{+}, \mathrm{S}^{+}$, and $\mathrm{Si}^{+}$), and electrons. For the purposes of an accurate calculation of the equilibrium abundances of charged particles, we also include (negative) singly charged grains and neutral grains. However, the collisional effects of grains on the neutrals (which in certain cases can be significant; see CM94, CM95) are ignored in this paper: this is done by setting the parameter $\langle\widetilde{\sigma w}\rangle_{\mathrm{gn}}$ (see below) equal to 0 . The effects of magnetic braking and rotation, which can also affect the evolution of a core (Basu \& Mouschovias 1994, 1995a, b) are similarly neglected in this paper. They will be accounted for in a later publication.

The assumptions that model clouds are thin and that evolution along field lines is quasistatic allow the time-dependent nonlinear set of nonideal MHD equations that govern the evolution of the model cloud to be integrated over $z$, thus reducing the dimensionality of the formal problem (CM93). The simplified set of equations is listed as equations (A1)-(A17) in CM95. The equations are cast in dimensionless form by adopting the quantity $2 \pi G \sigma_{\mathrm{c}, \text { ref }}$ as the unit of acceleration, $B_{\text {ref }}$ as the magnetic field strength, and the isothermal speed of sound $C$ as the unit of velocity. The quantity $\sigma_{\text {c,ref }}$ is the central column density of a reference state used to specify how magnetic field lines are initially loaded with mass, and $B_{\text {ref }}$, as mentioned in $\S 2.2$, is the background magnetic field strength at infinity. The implied units of time, length, and mass density are, respectively, $C / 2 \pi G \sigma_{\mathrm{c}, \mathrm{ref}}$, $C^{2} / 2 \pi G \sigma_{\mathrm{c} \text {,ref }}$, and $2 \pi G \sigma_{\mathrm{c}, \mathrm{ref}}^{2} / C^{2}$. The resulting system of dimensionless equations contains the following set of nondimensional parameters:

$$
\begin{aligned}
\mu_{\mathrm{d}, \mathrm{c} 0} & \equiv \frac{\left(d M / d \Phi_{B}\right)_{\mathrm{c} 0}}{\left(d M / d \Phi_{B}\right)_{\mathrm{d}, \mathrm{crit}}}=\frac{\left(\sigma_{\mathrm{c}, \mathrm{ref}} / B_{\mathrm{ref}}\right)}{(1 / 2 \pi \sqrt{G})}=0.196\left(\frac{\sigma_{\mathrm{c}, \mathrm{ref}}}{3.63 \times 10^{-3} \mathrm{~g} \mathrm{~cm}^{-2}}\right)\left(\frac{30 \mu \mathrm{G}}{B_{\mathrm{ref}}}\right), \\
\tilde{P}_{\mathrm{ext}} & \equiv \frac{P_{\mathrm{ext}}}{(\pi / 2) G \sigma_{\mathrm{c}, \mathrm{ref}}^{2}}=0.1\left(\frac{P_{\mathrm{ext}}}{1.38 \times 10^{-13}{\mathrm{dyn} \mathrm{cm}^{-2}}}\right)\left(\frac{3.63 \times 10^{-3} \mathrm{~g} \mathrm{~cm}^{-2}}{\sigma_{\mathrm{c}, \mathrm{ref}}}\right),
\end{aligned}
$$




$$
\begin{aligned}
\langle\widetilde{\sigma w}\rangle_{\mathrm{iH}_{2}} & \equiv \frac{\langle\sigma w\rangle_{\mathrm{iH}_{2}}}{1.4\left[1+\left(m_{\mathrm{H}_{2}} / m_{\mathrm{i}}\right)\right]} \frac{\left(2 \pi G \sigma_{\mathrm{c}, \mathrm{ref}}\right)^{2}}{C^{5}} \\
& =1.2 \times 10^{-48} \frac{\left(\langle\sigma w\rangle_{\mathrm{iH}} / 1.7 \times 10^{-9} \mathrm{~cm}^{3} \mathrm{~s}^{-1}\right)}{1+\left[15\left(m_{\mathrm{i}} / m_{\mathrm{H}_{2}}\right)\right]^{-1}}\left(\frac{\sigma_{\mathrm{c}, \mathrm{ref}}}{3.63 \times 10^{-3} \mathrm{~g} \mathrm{~cm}^{-2}}\right)^{2}\left(\frac{0.188 \mathrm{~km} \mathrm{~s}^{-1}}{C}\right)^{5} \\
\langle\widetilde{\sigma w}\rangle_{\mathrm{gn}} & \equiv\langle\sigma w\rangle_{\mathrm{gn}} \frac{\left(2 \pi G \sigma_{\mathrm{c}, \mathrm{ref}}\right)^{2}}{C^{5}} \\
& =9.3 \times 10^{-47}\left(\frac{a}{10^{-6} \mathrm{~cm}}\right)^{2}\left(\frac{\sigma_{\mathrm{c}, \mathrm{ref}}}{3.63 \times 10^{-3} \mathrm{~g} \mathrm{~cm}^{-2}}\right)^{2}\left(\frac{0.188 \mathrm{~km} \mathrm{~s}^{-1}}{C}\right)^{4}
\end{aligned}
$$

where the quantity $a$ in equation (A4) is the grain radius. The parameter $\mu_{\mathrm{d}, \mathrm{c} 0}$ is the initial central mass-to-flux ratio in units of the critical value for collapse. $\tilde{P}_{\text {ext }}$ is the constant external pressure normalized to the self-gravitational stress of the matter contained within the central flux tube of the reference state. The parameter $\langle\widetilde{\sigma w}\rangle_{\mathrm{iH}_{2}}$ is the dimensionless neutral-ion collisional rate, and $\langle\widetilde{\sigma w}\rangle_{\text {gn }}$ is the dimensionless neutral-grain collisional rate. The initial equilibrium state introduces one more parameter, the quantity $\tilde{l}_{\text {ref }}$, a length scale in the column density of the reference state (see CM93, eqs. [60b] and [73b]). The dimensionless cloud radius is taken to be $5 \tilde{l}_{\text {ref }}$ in this paper. A final dimensionless parameter is the initial dust-to-gas mass ratio, $\chi_{\mathrm{g}, 0}$, which is equal to 0.01 in the typical model.

Abundances of charged particles are determined by solution of the chemical rate equations with balance between creation and destruction of the various charged species specified above, accounting for such processes as cosmic-ray ionization, dissociative recombination of molecular ions and electrons, radiative recombination of atomic ions and electrons, attachment of charged particles on grains, and charge transfer between molecular and atomic ions; we also account for ionization due to an external (interstellar) ultraviolet radiation field. The relevant chemical equations are given by equations (7)-(17) of CM95. They contain six relevant nondimensional parameters of the form $\zeta_{\alpha_{0}, \mathrm{UV}, \mathrm{CR}} \equiv \zeta_{\alpha_{0}, \mathrm{UV}} / \zeta_{\mathrm{CR}}$, where $\zeta_{\alpha_{0}}$ is the UV ionization rate at the cloud boundary for neutral species $\alpha_{0}$, and $\zeta_{\mathrm{CR}}$ is the cosmic-ray ionization rate.

\section{APPENDIX B}

\section{DENSITY SCALING IN THE INNER FLUX TUBES OF MODEL CLOUDS}

For our thin-disk model, the density $\rho_{\mathrm{n}}\left(=m_{\mathrm{n}} n_{\mathrm{n}}\right)$ is calculated from equation (9). We examine the power-law behavior of the density in the inner flux tubes of the core for the case in which the tidal gravitational field of the central protostar is negligible, and then for 
the opposite case where it is the dominant term in equation (9). In the first case, using the fact that $P_{\text {ext }} \ll(\pi / 2) G \sigma_{\mathrm{n}}^{2}$ in the inner flux tubes of the core and that the magnetic squeezing term is also negligible there for most times of interest (see discussion in $\S 2.2$ following eq. [9]), the above equation yields $\rho_{\mathrm{n}}=\pi G \sigma_{\mathrm{n}}^{2} / 2 C^{2}$. During the later stages of the pre-PMF phase of core contraction, $\sigma_{\mathrm{n}} \propto r^{-1}$ (CM94, CM95; Basu \& Mouschovias 1994, 1995a,b; see also $\S 3.2)$. Therefore $\rho_{\mathrm{n}} \propto r^{-2}$ as the core approaches PMF.

We now turn to the case where the tidal stress from the central protostar is the dominant term on the r.h.s. of equation (9). Near the protostar, tidal squeezing of the disk (see Fig. $6 i$ ) results in $Z / r=\sigma_{\mathrm{n}} / 2 \rho_{\mathrm{n}} r \ll 1$. 㡄 Expansion of the second term in braces on the r.h.s. of equation (9) gives the solution $\rho_{\mathrm{n}}=\left(G M_{\text {cent }} / 8\right)^{1 / 2} \sigma_{\mathrm{n}} / C r^{3 / 2}$. In the limit of free-fall collapse near the protostar $\sigma_{\mathrm{n}} \propto r^{-1 / 2}($ see $\S 3.3)$ and $\rho_{\mathrm{n}}$ is again $\propto r^{-2}$. Hence, the power-law behavior of the density in our model is constrained to be $\propto r^{-2}$ before and after PMF. This result is different from the behavior $\rho_{\mathrm{n}} \propto r^{-3 / 2}$, which would occur for the case of spherical infall in the gravitational field of a central point mass. This difference is due to our use of the vertical one-zone approximation for the mass density $\left[\rho_{\mathrm{n}}(z, r, t)=\rho_{\mathrm{n}}(0, r, t) \equiv \rho_{\mathrm{n}}(r, t)\right]$ in equation (9) for balance of forces along field lines. Figure 9 shows the number density $n_{\mathrm{n}}$, normalized to $n_{\mathrm{n}, \mathrm{c} 0}=2.6 \times 10^{3} \mathrm{~cm}^{-3}$, as a function of $r / R_{0}$ at the same seven times $\Delta t_{j}$ as in Figure 6. The $r^{-2}$ scaling of the density is apparent in this figure at small radii.

\section{APPENDIX C}

\section{BREAKDOWN OF FLUX FREEZING AFTER POINT-MASS FORMATION}

In this Appendix we present a heuristic argument that demonstrates the breakdown of flux freezing, and the corresponding revitalization of ambipolar diffusion, following point-mass formation (PMF). We show this by means of a reductio ad absurdum argument: we assume that the collapse proceeds under flux freezing conditions, and we use the frozen-flux solution to evaluate the ratio of the ion-neutral drift speed $\left(v_{\mathrm{D}}\right)$ and the free-fall speed $\left(v_{\mathrm{ff}}=[2 G M(r) / r]^{1 / 2}\right)$. Flux freezing corresponds to $v_{\mathrm{D}} \ll v_{\mathrm{ff}}$; however, by deriving the magnetic force on the ions from the numerically computed evolution and using the ion equation of motion to calculate the resulting ion-neutral drift speed, we find that the ratio $v_{\mathrm{D}} / v_{\mathrm{ff}}$ becomes $\gg 1$ after PMF. This implies that the assumption of flux freezing is not self-consistent and that ambipolar diffusion necessarily sets in. According to this

\footnotetext{
${ }^{14}$ This squeezing will be augmented, particularly at late times, by magnetic field effects represented by the second term on the r.h.s. of equation (9).
} 
argument, the increase of $v_{\mathrm{D}} / v_{\mathrm{ff}}$ in the inner core following PMF can be attributed to the growth of the magnetic tension term in the ion force equation. The quantity $v_{\mathrm{D}} / v_{\mathrm{ff}}$ is essentially the inverse of the ratio of the ambipolar diffusion timescale $\left(\tau_{\mathrm{AD}} \equiv r / v_{\mathrm{D}}\right)$ and gravitational contraction timescale $\left[\tau_{\mathrm{gr}} \equiv\left(r /\left|g_{r}\right|\right)^{1 / 2} \approx \tau_{\text {ff }}\right]$ considered in $\S 3.3$ (see eq. [15]). In the argument presented in $\S 3.3$ (see also $\S 1$ ), $\tau_{\mathrm{AD}} / \tau_{\mathrm{gr}}$ is shown to decrease below 1 during that epoch on account of the effect of the central point mass on the magnitude of the gravitational acceleration in its vicinity. As we show below, these two alternative descriptions of the ambipolar-diffusion revitalization process are equivalent.

In a disk-like cloud, the dominant terms in the expression for the radial magnetic force per unit mass are

$$
\begin{aligned}
\frac{F_{\mathrm{mag}, r}}{\sigma_{\mathrm{n}}} & =\frac{1}{2 \pi \sigma_{\mathrm{n}}}\left(B_{z, \mathrm{eq}} B_{r, Z}-Z \frac{\partial B_{z, \mathrm{eq}}}{\partial r}\right) \\
& =\frac{B_{z, \mathrm{eq}} B_{r, Z}}{2 \pi \sigma_{\mathrm{n}}}\left(1-\frac{Z}{B_{z, \mathrm{eq}}} \frac{\partial B_{z, \mathrm{eq}}}{\partial r}\right)
\end{aligned}
$$

(see eq. [28c] of Ciolek \& Mouschovias 1993). The first term on the r.h.s. of equation (C5) is the magnetic tension force, while the second term is the magnetic pressure force. It turns out (see below) that, in a frozen-flux core, the tension term comes to dominate after PMF. In that case, using the ion force equation

$$
\frac{\sigma_{\mathrm{n}}}{\tau_{\mathrm{ni}}} v_{\mathrm{D}}=F_{\mathrm{mag}, r}
$$

and the continuity equation $\left(\dot{M}=-2 \pi r \sigma_{\mathrm{n}} v_{\mathrm{n}}\right)$, and assuming $\left|v_{\mathrm{n}}\right| \approx v_{\mathrm{ff}}$ in the inner flux tubes of a collapsing core, one obtains

$$
\frac{v_{\mathrm{D}}}{v_{\mathrm{ff}}}=\left[\frac{\tau_{\mathrm{ni}}}{2 \mu_{B}^{2}} \frac{\dot{M}}{M}\right] \frac{B_{r, Z}}{B_{z, \mathrm{eq}}},
$$

where $\mu_{B}$ is the mass-to-flux ratio in units of the critical value for collapse, as discussed in $\S 1$. The term in brackets on the r.h.s. of equation (C7) is the same as equation (4) of Li \& McKee (1996), who used $\epsilon=\mu_{B}^{-1}$ in their expression. Consider now the behavior of $B_{r, Z}$ and $B_{z \text {,eq }}$ in a frozen-flux core following PMF. As was noted in $\S 2.2, B_{z, \text { eq }} \propto \sigma_{\mathrm{n}}$ and $B_{r, Z} \propto g_{r}$ in a thin, perfectly conducting disk with $\mu_{B}=$ const. Thus, outside the central sink cell, $B_{r, Z} \approx \Phi_{B}(r, t) / 2 \pi r^{2} \approx \Phi_{B \text {,cent }} / 2 \pi r^{2}$ after PMF (see eq. [8] and associated discussion). Here $\Phi_{B}(r, t)$ is the total flux enclosed within radius $r$, which is $\sim \Phi_{B \text {, cent }}$ after PMF. Hence, $B_{r, Z}$ scales as $r^{-2}$. Near the central point mass the inflowing matter is in approximate free fall and hence $B_{z, \text { eq }} \propto \sigma_{\mathrm{n}} \propto r^{-1 / 2}$ (see $\S 3.3$ ). Therefore, after PMF, $B_{r, Z} / B_{z, \text { eq }} \propto \Phi_{B, \text { cent }} / r^{3 / 2}$ will become $\gg 1$ as $r \rightarrow 0$. This behavior has indeed been seen 
in our frozen-flux model simulations, and is also apparent in the self-similar frozen-field collapse calculations of Li \& Shu (1997) and CCK.

Because of the increase of $B_{r, Z} / B_{z \text {,eq }}$ near the origin after $\mathrm{PMF}$, the ratio $v_{\mathrm{D}} / v_{\mathrm{ff}}$ given by equation (C7) will also become $\gg 1$ there. This is illustrated graphically in Figures $10 a$, $10 b$, and $10 c$, which show, respectively, the ratios $v_{\mathrm{D}} /\left|v_{\mathrm{n}}\right|, B_{r, Z} / B_{z \text {,eq }}$, and $F_{\text {mag, } r} / F_{\text {mag,pres }}$ in the inner flux tubes of the frozen-flux model core presented in $\S 3.3$. The quantity $F_{\operatorname{mag}, r}$ is the actual magnetic force per unit mass calculated in our model (given by eq. [C5 above), which includes magnetic tension, whereas $F_{\text {mag,pres }}=B_{z \text {,eq }}^{2} / 2 \pi \sigma_{\mathrm{n}}$ is the force term used by Li \& McKee (1996), which approximately represents the effect of magnetic pressure alone. It is seen from these figures that $v_{\mathrm{D}} /\left|v_{\mathrm{n}}\right|$ does indeed become $\gg 1$ as the core evolves, and that this is due to the increase of $B_{r, Z} / B_{z \text {,eq }}$, which scales as $r^{-3 / 2}$, as expected. (As pointed out in $\S 3.3$, our frozen-flux model is calculated by setting $v_{\mathrm{i}}=v_{\mathrm{n}}$, so that $v_{\mathrm{D}}=0$ in our numerical code; to obtain $v_{\mathrm{D}}$ in Fig. 10a, we used equations [C6] and [C5].) These results also confirm that $F_{\text {mag, } r} / F_{\text {mag,pres }}=B_{r, Z} / B_{z, \text { eq }}$. The fact that $v_{\mathrm{D}} /\left|v_{\mathrm{n}}\right|$ becomes $\gg 1$ means that the assumption of continued flux freezing is not self-consistent and that ambipolar diffusion must eventually take place. 15 The region of flux decoupling will move outward with time, which is consistent with the fact that ambipolar diffusion in our simulations first becomes noticeable on our smallest resolvable scale, and that the decoupling front subsequently moves outward.

This " $B_{r, Z} / B_{z, \text { eq }}$ " explanation of the onset of ambipolar diffusion is the same as the " $\tau_{\mathrm{gr}} / \tau_{\mathrm{ni}}$ " argument given in $\S \S 1$ and 3.3, where we emphasized the fact that $\tau_{\mathrm{AD}} / \tau_{\mathrm{gr}} \sim \tau_{\mathrm{gr}} / \tau_{\mathrm{ni}}$. This can be seen in the following way. Substituting $B_{r, Z} \approx \Phi_{B}(r, t) / 2 \pi r^{2}$ into the ion force equation (C6), we get

$$
v_{\mathrm{D}}=\frac{\tau_{\mathrm{ni}}}{2 \pi \sigma_{\mathrm{n}}} B_{z, \mathrm{eq}} \frac{\Phi_{B}}{2 \pi r^{2}} .
$$

Dividing this equation by $v_{\mathrm{ff}}$, and using the relation $M=v_{\mathrm{ff}}^{2} r / 2 G$, yields

$$
\frac{v_{\mathrm{D}}}{v_{\mathrm{ff}}}=\tau_{\mathrm{ni}}\left[\frac{B_{z, \mathrm{eq}}}{4 \pi^{2} G \sigma_{\mathrm{n}}} \frac{\Phi_{B}}{M}\right] \frac{v_{\mathrm{ff}}}{2 r}=\frac{1}{\sqrt{2} \mu_{B}^{2}} \frac{\tau_{\mathrm{ni}}}{\tau_{\mathrm{gr}}},
$$

where, in the last equality, we used $\tau_{\mathrm{gr}}=\sqrt{2} r / v_{\mathrm{ff}}$ and the definition of $\mu_{B}$. From this equation it follows that $\tau_{\mathrm{AD}} / \tau_{\mathrm{gr}} \approx v_{\mathrm{ff}} / v_{\mathrm{D}}=\mu_{B}^{2} \tau_{\mathrm{gr}} / \tau_{\mathrm{ni}}$. The expression given by equation

\footnotetext{
${ }^{15}$ This conclusion did not follow from the estimate of $v_{\mathrm{D}} / v_{\mathrm{ff}}$ in Li \& McKee (1996) because they took the radial magnetic force per unit mass to be $F_{\text {mag,pres }}$ rather than $F_{\text {mag, } r}$ and thus omitted the ratio $B_{r, Z} / B_{z \text {,eq }}$ from their equation (4). The argument presented in this Appendix also explains why the revitalization of ambipolar diffusion following PMF cannot be studied using a spherically symmetric model (e.g., Li 1998), where only the magnetic pressure force, but not the tension force, is taken into account.
} 
(C9) is the same as that derived by Mouschovias (1991, eq. [10a]), who found that $\tau_{\mathrm{AD}} \propto\left(\tau_{\mathrm{gr}}^{2} / \tau_{\mathrm{ni}}\right)\left(\Phi_{B, \text { crit }} / \Phi_{B}\right)^{2}$, where $\Phi_{B, \text { crit }}$ is the critical magnetic flux. Finally, by inserting equations (C6) and (C8) into equation (14) and using the relations $\left|g_{r}\right| \approx G M / r^{2}$ and $B_{r, Z} \approx \Phi_{B} / 2 \pi r^{2}$ in the central flux tubes of a core following PMF (see eqs. [6] and [8]), one obtains (neglecting thermal-pressure forces)

$$
\left(1-\frac{\left|a_{\mathrm{n}}\right|}{\left|g_{r}\right|}\right)=\left(1-\frac{\tau_{\mathrm{gr}}^{2}}{\tau_{\mathrm{acc}}^{2}}\right)=\frac{\left(B_{z, \mathrm{eq}} / \sigma_{\mathrm{n}}\right)\left(\Phi_{B} / M\right)}{4 \pi^{2} G}=\mu_{B}^{-2} .
$$

(This relation can also be derived from eq. [24] of Basu 1997.) Therefore, equations (C9) and (C10) yield equation (15), and the " $B_{r, Z} / B_{z, \text { eq }}$ " and " $\tau_{\mathrm{gr}} / \tau_{\mathrm{ni}}$ " pictures are seen to be completely equivalent, as claimed. 


\section{TABLE 1 \\ Dimensionless Parameters of a Typical Model Cloud ${ }^{\dagger}$}

\begin{tabular}{ccccc}
\hline \hline$\mu_{\mathrm{d}, \mathrm{c} 0}$ & $\tilde{l}_{\mathrm{ref}}$ & $\tilde{P}_{\mathrm{ext}}$ & $\langle\widetilde{\sigma w}\rangle_{\mathrm{iH}_{2}}$ & $\langle\widetilde{\sigma w}\rangle_{\mathrm{gn}}$ \\
\hline 0.256 & $5.5 \pi$ & 0.1 & $2.53 \times 10^{-48}$ & 0 \\
\hline \hline
\end{tabular}

${ }^{\dagger}$ For a discussion of the meaning of these parameters, see Appendix A.

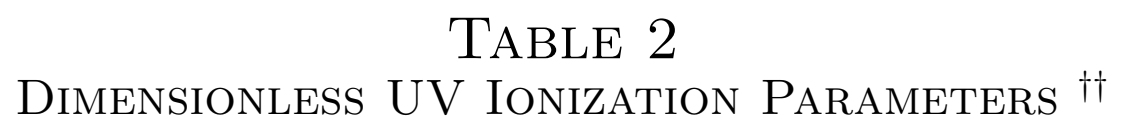

\begin{tabular}{cccccc}
\hline \hline$\zeta_{\mathrm{C}_{0}, \mathrm{UV}, \mathrm{CR}}$ & $\zeta_{\mathrm{Mg}_{0}, \mathrm{UV}, \mathrm{CR}}$ & $\zeta_{\mathrm{Na}_{0}, \mathrm{UV}, \mathrm{CR}}$ & $\zeta_{\mathrm{Fe}_{0}, \mathrm{UV}, \mathrm{CR}}$ & $\zeta_{\mathrm{S}_{0}, \mathrm{UV}, \mathrm{CR}}$ & $\zeta_{\mathrm{Si}_{0}, \mathrm{UV}, \mathrm{CR}}$ \\
$\left(10^{6}\right)$ & $\left(10^{6}\right)$ & $\left(10^{6}\right)$ & $\left(10^{6}\right)$ & $\left(10^{6}\right)$ & $\left(10^{6}\right)$ \\
\hline 2.60 & 0.90 & 0.11 & 2.40 & 14.4 & 24.0 \\
\hline \hline
\end{tabular}

it All ionization rates are normalized to a cosmic-ray ionization rate of $5 \times 10^{-17} \mathrm{~s}^{-1}$. All dimensional UV ionization rates are taken from Table 9 of Black \& Dalgarno (1977, and references therein). 


\section{REFERENCES}

André, P. 1995, Ap\&SS, 224, 29

Bachiller, R., Tafalla, M., \& Cernicharo, J 1994, ApJ, 425, L93

Basu, S. 1997, ApJ, 485, 240

Basu, S., \& Mouschovias, T. Ch. 1994, ApJ, 432, 720

. 1995a, ApJ, 452, 386 . 1995b, ApJ, 453, 271

Black, J. H., \& Dalgarno, A. 1977, ApJS, 34, 405

Blaes, O. M., \& Balbus, S. A. 1994, ApJ, 421, 163

Bontemps, S., André, P., Terebey, S., \& Cabrit, S. 1996, A\&A, 311, 858

Boss, A. P. 1984, ApJ, 277, 768

Boss, A. P., \& Black, D. C. 1982, ApJ, 258, 270

Carpenter, J. M., Snell R. L., Schloerb, F. P., \& Skrutskie, M. F. 1993, ApJ, 407, 657

Chernoff, D. F. 1987, ApJ, 312, 143

Chiueh, T., \& Chou, J.-K. 1994, ApJ, 431, 380

Ciolek, G. E. 1993, Ph.D. thesis, Univ. of Illinois

Ciolek, G. E., \& Mouschovias, T. Ch. 1993, ApJ, 418, 774 (CM93) . 1994, ApJ, 425, 142 (CM94) . 1995, ApJ, 454, 194 (CM95)

Contopolous, I., Ciolek, G. E., \& Königl, A. 1997, ApJ, submitted (CCK97)

Crutcher, R. M., Mouschovias, T. Ch., Troland, T. H., \& Ciolek, G. E. 1994, ApJ, 427, 839

Crutcher, R. M., Roberts, D. A., Mehringer, D. M., \& Troland, T. H. 1996, ApJ, 462, L79

Crutcher, R. M., Troland, T. H., Goodman, A. A., Heiles, C., Kazés, I., \& Myers, P. C. 1993, ApJ, 407, 175

Draine, B. T. 1980, ApJ, 241, 1021 . 1986, MNRAS, 220, 133

Draine, B. T., \& McKee, C. F. 1993, ARA\&A, 31, 373

Fiedler, R. A., \& Mouschovias, T. Ch. 1992, ApJ, 391, 199 . 1993, ApJ, 415, 680 
Foster, P. C., \& Chevalier, R. A. 1993, ApJ, 416, 303

Gaustad, J. E. 1963, ApJ, 138, 1050

Gioumousis, G., \& Stevenson, D. P. 1958, J. Chem. Phys., 29, 294

Greaves, J. S., Holland, W. S., \& Murray, A. G. 1995, MNRAS, 297, L49

Greaves, J. S., Murray, A. G., \& Holland, W. S. 1994, MNRAS, 284, L19

Hayashi, C. 1966, ARA\&A, 4, 171

Hayashi, M., Ohashi, N., \& Miyamam, S. M. 1993, ApJ, 418, L71

Hildebrand, R. H. 1989, in Interstellar Dust, IAU Symposium No. 135, ed. L. J. Allamandola \& A. G. G. M. Tielens (Dordrecht: Kluwer), 275 . 1996, in Polarimetry of the Interstellar Medium, ed. W. G. Roberge \& D. C. B. Whittet, ASP Conf. Series, 97, 254

Hildebrand, R. H., Dotson, J. L., Dowell, C. D., Platt, S. R., Schleuning, D., Davidson, J. A., \& Novak, G. 1995, in Airborne Astronomy Symposium on the Galactic Ecosystem, ed. M. R. Haas, J. A. Davidson, \& E. F. Erickson, ASP Conf. Series, 73, 97

Hildebrand, R. H., Dragovan, M., \& Novak, G. 1984, ApJ, 284, L51

Hunter, C. 1977, ApJ, 218, 834

Kane, B. D., Clemens, D. P., Barvainis, R., \& Leach, R. W. 1993, ApJ, 411, 708

Königl, A. 1989, ApJ, 342, 208

Königl, A., \& Ruden, S. 1993, in Protostars and Planets III, ed. E. H. Levy \& J. I. Lunine (Tucson: Univ. Arizona Press), 641

Ladd, E. F., Myers, P. C., \& Goodman, A. A. 1994, in Clouds, Cores, and Low Mass Stars, ed. D. P. Clemens \& R. Barvainis, ASP Conf. Series, 65, 19

Larson, R. B. 1969, MNRAS, 145, 271 . 1972, MNRAS, 145, 271

Li, Z.-Y. 1996, ApJ, 465, 855 . 1998, ApJ, 493, 230

Li, Z.-Y., \& McKee, C. F. 1996, ApJ, 464, 373

Li, Z.-Y., \& Shu, F. H. 1997, ApJ, 475, 237

Lizano, S., \& Shu, F. H. 1989, ApJ, 342, 834

Lubow, S. H., Papaloizou, J. C. B., \& Pringle, J. E. 1994, MNRAS, 267, 235 
Lubow, S. H., \& Spruit, H. C. 1995, ApJ, 445, 337

McDaniel, E. W., \& Mason, E. A. 1973, in The Mobility and Diffusion of Ions and Gases (New York: Wiley)

McKee, C. F., Zweibel, E. G., Goodman, A. A., \& Heiles, C. 1993, in Protostars and Planets III, ed. E. H. Levy \& J. I. Lunine (Tucson: Univ. of Arizona Press), 327

Mestel, L., \& Spitzer, L., Jr. 1956, MNRAS, 187, 116, 503

Morton, S. A., Mouschovias, T. Ch., \& Ciolek, G. E. 1994, ApJ, 421, 561 (MMC94)

Mouschovias, T. Ch. 1978, in Protostars and Planets, ed. T. Gehrels (Tucson: Univ. Arizona Press), 209

. 1987, in Physical Processes in Interstellar Clouds, ed. G. E. Morfill \& M. Scholer (Dordrecht: Reidel) 453

. 1989, in The Physics and Chemistry of Interstellar Clouds, ed. G. Winnewisser \& J. T. Armstrong (Berlin: Springer-Verlag), 297

. 1991, ApJ, 373, 169

. 1996, in Solar and Astrophysical Magnetohydrodynamic Flows, ed. K. Tsinganos (Dordrecht: Kluwer), 447

Mouschovias, T. Ch., \& Morton, S. A. 1991, ApJ, 371, 296

Mouschovias, T. Ch., \& Paleologou, E. V. 1981, ApJ, 246, 48

Mouschovias, T. Ch., Paleologou, E. V., \& Fiedler, R. A. 1985, ApJ, 291, 772

Mullan, D. J. 1971, MNRAS, 153, 145

Myhill, E. A., \& Boss, A. P. 1993, ApJS, 89, 345

Myhill, E. A., \& Kaula, W. M. 1992, ApJ, 386, 578

Nakano, T. 1984, Fund. Cos. Phys., 9, 139

Nakano, T., \& Nakamura, T. 1978, PASJ, 30, 671

Nakano, T., \& Umebayashi, T. 1986a, MNRAS, 218, 663 . 1986b, MNRAS, 221, 319

Norman, C., \& Heyvaerts, J. 1985, A\&A, 147, 247

Novak, G., Gonatas, D. P., Hildebrand, R. H., Platt, S. R., \& Dragovan, M. 1989, ApJ, 345,802

Ohashi, N., Hayashi, M., Ho, P. T. P., Momose, M., \& Hirano, N 1996, ApJ, 466, 957

Paleologou, E. V., \& Mouschovias, T. Ch. 1983, ApJ, 275, 838 
Penston, M. V. 1969, MNRAS, 144, 425

Pneuman, G. W., \& Mitchell, T. P. 1965, Icarus, 4, 494

Reyes-Ruiz, M., \& Stepinski, T. F. 1996, ApJ, 459, 653

Roberge, W. G., \& Draine, B. T. 1990, ApJ, 350, 700

Ruffle, D. P., Hartquist, T. W., Rawlings, J. M. C., \& Williams, D. A. 1998, A\&A, submitted

Safier, P. N., McKee, C. F., \& Stahler, S. W. 1997, ApJ, 485, 660

Saraceno, P., André, P., Ceccaralli, C., Griffin, M., \& Molinari, S. 1996, A\&A, 309, 827

Schleuning, D. A. 1998, ApJ, in press

Shu, F. H. 1977, ApJ, 214, 488

Shu, F. H., Adams, F. C., \& Lizano, S. 1987, ARA\&A, 25, 33

Smith, M. D., \& Mac Low, M.-M. 1997, A\&A, in press

Spitzer, L. Jr. 1963, in Origin of the Solar System, ed. R. Jastrow \& A. G. W. Cameron (New York: Academic), 39

Spruit, H. C., Stehle, R., \& Papaloizou, J. C. B. 1995, MNRAS, 275, 1223

Spruit, H. C., \& Taam, R. E. 1990, ApJ, 475, 229

Stahler, S. W., Shu, F. H., \& Taam, R. E. 1981, ApJ, 248, 727

Tomisaka, K 1996, PASJ, 48, L97

Ward-Thompson, D. 1996, Ap\&SS, 239, 151

Wardle, M. 1990, MNRAS, 246, 98

Wardle, M., \& Königl, A. 1993, ApJ, 410, 218

Wardle, M., \& Ng, C. 1998, MNRAS, submitted

Whitworth, A. P., \& Summers, D. 1985, MNRAS, 214, 1

Winkler, K. H., Newman, M. J. 1980, ApJ, 236, 201

Zweibel, E. G., \& Lovelace, R. V. E. 1997, ApJ, 475, 260 


\section{Figure Captions}

Fig. 1.- Spatial profiles of physical quantities in the typical model, prior to point-mass formation, as functions of radius $r$ (normalized to the initial cloud radius $R_{0}$ $=4.29 \mathrm{pc})$ at eleven different times $t_{j}(j=0,1,2, \ldots, 10)$ chosen such that the central density has increased by a factor of $10^{j}$ with respect to its initial value. These times are 0, 7.56 Myr, 9.29 Myr, 9.56 Myr, 9.607 Myr, 9.6167 Myr, 9.6169 Myr, 9.6190 Myr, 9.61977 Myr, 9.61981 Myr, and 9.61983 Myr, respectively. An asterisk on a curve, present only when a supercritical core has formed, marks the instantaneous position of the critical flux tube. An open circle on every curve locates the instantaneous position of the critical thermal lengthscale. As discussed in $\S 2.2$, the assumption of isothermality and the form of the induction equation used in our calculation are not valid for densities $n_{\mathrm{n}}>10^{11} \mathrm{~cm}^{-3} \approx 4 \times 10^{7} n_{\mathrm{n}, \mathrm{c} 0}$. (a) Neutral density, normalized to its initial central value $n_{\mathrm{n}, \mathrm{c} 0}\left(=2.6 \times 10^{3} \mathrm{~cm}^{-3}\right)$. (b) Neutral column density, normalized to its initial central value $\sigma_{\mathrm{n}, \mathrm{c} 0}\left(=5.59 \times 10^{-3} \mathrm{~g} \mathrm{~cm}^{-2}\right)$. (c) Mass-to-flux ratio, in units of the critical value for gravitational collapse. Between times $t_{1}$ and $t_{2}$ a critical core has formed (because of ambipolar diffusion) inside a magnetically subcritical envelope. $(d)$ Vertical $(z)$ component of the magnetic field in the equatorial plane of the disk, normalized to its initial central value $B_{z, \mathrm{eq}, \mathrm{c} 0}(=35.3 \mu \mathrm{G})$. (e) Radial $(r)$ component of the magnetic field at the upper surface of the cloud, normalized to $B_{z, \mathrm{eq}, \mathrm{c} 0} .(f)$ Infall speed of the neutrals, normalized to the isothermal speed of sound $C\left(=0.19 \mathrm{~km} \mathrm{~s}^{-1}\right)$. By the end of the run, $\left|v_{\mathrm{n}}\right| \approx C$ in the inner core. ( $g$ ) Ion-neutral drift speed ( $\equiv v_{\mathrm{i}}-v_{\mathrm{n}}$ ), normalized as in $(f)$. Ion depletion at higher densities is responsible for the appearance of the maximum in the core for $t \geq t_{6}$. $(h)$ Mass infall rate, in units of $M_{\odot} \mathrm{Myr}^{-1}$. (i) Ratio of cloud vertical half-thickness $Z(r)$ and radius $r$.

Fig. 2.- Accretion in the central cell during the PMF epoch. (a) Central (protostellar) mass, in $M_{\odot}$, as a function of time $\Delta t\left(=t-t_{10}\right)$. Formation of a point mass occurs at $\Delta t \lesssim 200 \mathrm{yr}$. (b) Accretion rate in $M_{\odot} \mathrm{Myr}^{-1}$. Dashed curve refers to a model that did not include the effect of ambipolar diffusion for $\Delta t \geq 0$. For $\Delta t \gtrsim 4 \times 10^{3} \mathrm{yr}$, ambipolar diffusion, through the formation of a hydromagnetic shock in the neutrals, decreases the protostellar accretion rate of the typical model. (c) Accretion rate in $M_{\odot} \mathrm{Myr}^{-1}$ as a function of central mass (in $M_{\odot}$ ). Dashed curve refers to the frozen-flux model, as in (b).

Fig. 3.- Physical quantities in two different computational mesh cells, as functions of $\Delta t$

( $\approx$ the time elapsed since PMF). (a) Cell 1: ratio of ambipolar-diffusion timescale $\tau_{\mathrm{AD}}$ and gravitational contraction timescale $\tau_{\mathrm{gr}}$, ratio of gravitational timescale and 
neutral-ion collision timescale $\tau_{\text {ni }}$, and (normalized) magnetic flux $\Phi_{B}$ contained within the cell. The gravitational contraction timescale decreases because of an increase in the magnitude of the gravitational field associated with the central accreting protostar, which increases the rate of ambipolar diffusion in the inner flux tubes of the model cloud. For $\Delta t \gtrsim 10^{3} \mathrm{yr}$, ambipolar diffusion has become rapid enough to halt the growth of magnetic flux within this cell. (b) Same as (a), but for cell 5. The evolution is similar to that of cell 1 except that it exhibits a time delay, indicating that the region of piled-up magnetic flux is advancing outward from the center.

Fig. 4.- Physical quantities in computational mesh cells $l=2,5,10,15,20,25$, and 27, as functions of $\Delta t$. (a) Vertical $(z)$ component of the magnetic field in the equatorial plane of the disk, normalized to $B_{z, \mathrm{eq}, \mathrm{c} 0}$. Prior to the passage of the hydromagnetic disturbance (HMD), the magnetic field strength decreases because of inward advection of magnetic flux. At later times the magnetic field is increased by the compression induced by the outward-propagating HMD. (b) Column density, normalized to $\sigma_{\mathrm{n}, \mathrm{c} 0}$. In cells 2 and 5 the inflow of the neutrals is unaffected by the advancement of the HMD, and the column density decreases with time due to advection of matter into the central sink. In cells $l>8$ the combination of the enhanced field strength (due to the piled-up magnetic flux) and the increased collisional coupling between the ions and neutrals at larger radii eventually leads to a deceleration of the infalling neutrals and the formation of a hydromagnetic shock. The column density increases immediately after the passage of the shock front; at later times the neutrals diffuse through the shock and are reaccelerated to free fall, with $\sigma_{\mathrm{n}} \propto(\Delta t)^{s} \Delta t,-0.76 \lesssim s_{\Delta t} \lesssim-0.55$.

Fig. 5.- Outward motion of the hydromagnetic disturbance as a function of $\Delta t . \quad(a)$ Instantaneous position of the HMD, normalized to the cloud radius $R_{0}$. (b) Speed of the disturbance (in the protostellar rest frame), normalized to $C$. Early on, the expansion of the HMD is nonsteady. At later times, the speed of the disturbance is subsonic. (c) Mach number relative to the infalling neutrals just upstream of the disturbance. Note that the time intervals between successive data points in this figure are larger than those in Figs. 2-4, reflecting a time-averaging of the motion of the HMD. For this reason the steady oscillations seen in Figs. 2-4 at later times do not appear in this figure.

Fig. 6.- Spatial profiles of physical quantities in the typical model during the post-PMF epoch as functions of $r / R_{0}$. Seven different times are plotted: $\Delta t=0,2.17 \times 10^{2} \mathrm{yr}$, $6.11 \times 10^{2} \mathrm{yr}, 1.54 \times 10^{3} \mathrm{yr}, 3.81 \times 10^{3} \mathrm{yr}, 2.38 \times 10^{4} \mathrm{yr}$, and $1.48 \times 10^{5} \mathrm{yr}$. An asterisk on a curve locates, as in Fig. 1, the instantaneous position of the critical 
magnetic flux tube. (a) Mass, in $M_{\odot}$. The protostar first forms at $\Delta t \approx 0$. (b) Vertical component of the magnetic field in the equatorial plane, normalized to $B_{z, \text { eq,c } 0}$. The dashed curve refers to a model in which the magnetic flux was assumed to be frozen into the neutrals at all times after PMF, shown at the last plotted time $\left(\Delta t_{6}\right)$. The field strength is strongly amplified behind the expanding HMD for $\Delta t>\Delta t_{2}$. For $\Delta t_{5} \lesssim \Delta t \lesssim \Delta t_{6}$ the field strength at $r / R_{0} \lesssim 2.5 \times 10^{-4}$ is significantly reduced, reflecting the refreezing of magnetic flux into the infalling neutrals brought about by the greatly enhanced collisional coupling that occurs when $v_{\mathrm{D}}>v_{\mathrm{D}, \text { crit }}$. (c) Neutral column density, normalized to $\sigma_{\mathrm{n}, \mathrm{c} 0}$. The shock in the neutrals manifests itself by the enhancement and local maximum in the column density for $\Delta t \geq \Delta t_{4}$. Far behind the shock the neutrals establish free-fall collapse, and $\sigma_{\mathrm{n}} \propto r^{-1 / 2}$ for $r \lesssim 4 \times 10^{-5} R_{0}$. The dashed curve refers to the model in which magnetic flux was frozen into the neutrals, at the same time as in $(b) .(d)$ Radial component of the magnetic field at the surface of the cloud, normalized to $B_{z, \mathrm{eq}, \mathrm{c} 0}$. For $\Delta t>\Delta t_{2}$, ambipolar diffusion halts the inward advection of flux and further bending of the field lines. The dashed curve refers to the frozen-flux model, at the same time as in $(b)$. Continual inward advection of magnetic flux in the frozen-in model results in much greater bending of field lines than in the ambipolar-diffusion case. (e) Infall speed of the neutrals, normalized to $C$. The location of the hydromagnetic shock is marked by the abrupt transition from supersonic to subsonic inflow for $\Delta t>\Delta t_{4}$. Free-fall collapse is established far behind the shock, and $v_{\mathrm{n}} \propto r^{-1 / 2}$ for $r \lesssim 4 \times 10^{-5} R_{0}=35.5 \mathrm{AU}$. $(f)$ Ion-neutral drift speed, normalized as in $(e)$. In the region of rapid ambipolar diffusion behind the expanding hydromagnetic disturbance, $v_{\mathrm{D}} \approx\left|v_{\mathrm{n}}\right|$. (g) Accretion rate, in $M_{\odot} \mathrm{Myr}^{-1}$. For $\Delta t \gtrsim \Delta t_{4}$ the accretion rate is decreased behind the shock front. ( $h$ ) Ratio of neutral-ion collisional timescale and gravitational contraction timescale. Near the inner boundary this ratio is $\gg 1$, indicating efficient ambipolar diffusion. Further away from the center, the degree of ionization increases and the magnitude of the gravitational field decreases, resulting in $\tau_{\text {ni }} / \tau_{\text {gr }}<1$ and, therefore, a much better collisional coupling between the ion and neutral fluids. ( $i$ ) Ratio of cloud vertical half-thickness $Z(r)$ and radius $r$. By the time $\Delta t_{6}$ the tidal gravitational field of the central protostar has compressed the inner core to a disk that is geometrically thin. [Magnetic squeezing of the disk, not included in this calculation, further reduces $Z(r)$.]

Fig. 7.- Mass of the gas surrounding the central protostar in the typical model, in $M_{\odot}$, as a function of $r / R_{0}$. All labels and times $\Delta t_{j}$ are the same as in Fig. 6.

Fig. 8. - Spatial profiles of quantities related to the stability of the core of the typical model cloud with respect to magnetic interchange, as a function of $r / R_{0}$. All labels and times 
$\Delta t_{j}$ are the same as in Fig. 6. (a) Local mass-to-flux ratio, in units of the critical value for collapse. Unloading of mass from field lines due to rapid ambipolar diffusion behind the hydromagnetic disturbance results in positive values of $d\left(\ln \sigma_{\mathrm{n}} / B_{z, \text { eq }}\right) / d r$ inside the core at times $\Delta t>\Delta t_{1}$. (b) Product of the linear instability growth rate and the neutral-ion collision time at $\Delta t=\Delta t_{6}$ for the region of the core susceptible to magnetic interchange. Collisional coupling of the ions and neutrals is sufficient to allow the instability to grow in this region of the core. (c) Product of the linear instability growth rate and the kinematical timescale $\left(=r /\left|v_{n}\right|\right)$ for the same time and region of the core as in $(b)$. An unstable mode can grow before being advected downstream by the fluid.

Fig. 9.- Density profile of the typical model, normalized to $n_{\mathrm{n}, \mathrm{c} 0}$, as a function of $r / R_{0}$. All labels and times $\Delta t_{j}$ are the same as in Fig. 6. In the inner core, $n_{\mathrm{n}} \propto r^{-2}$ throughout the post-PMF epoch.

Fig. 10.- Physical quantities in the inner flux tubes of the frozen-flux model as functions of $r / R_{0}$ at seven different times $\Delta t_{j}$. (a) Ratio of ion-neutral drift speed and the neutral infall speed. In this model, $v_{\mathrm{i}}$ is set equal to $v_{\mathrm{n}}$ in the numerical code, and the drift speed $v_{\mathrm{D}}$ is calculated from the ion force equation. As the core evolves, $v_{\mathrm{D}} /\left|v_{\mathrm{n}}\right|$ becomes $\gg 1$, indicating that the freezing of magnetic flux in the neutrals would break down. (b) Ratio of the $r$ and $z$ components of the magnetic field. (c) Ratio of the total radial magnetic force and the magnetic pressure force. After PMF, the ratios $B_{r, Z} / B_{z \text {,eq }}$ and $F_{\text {mag,r }} / F_{\text {mag,pres }}$ scale as $r^{-3 / 2}$. The enhanced magnetic tension force following PMF would act to revitalize ambipolar diffusion during this phase of the collapse, which is indeed what is found to occur in the typical model presented in $\S 3.3$. 

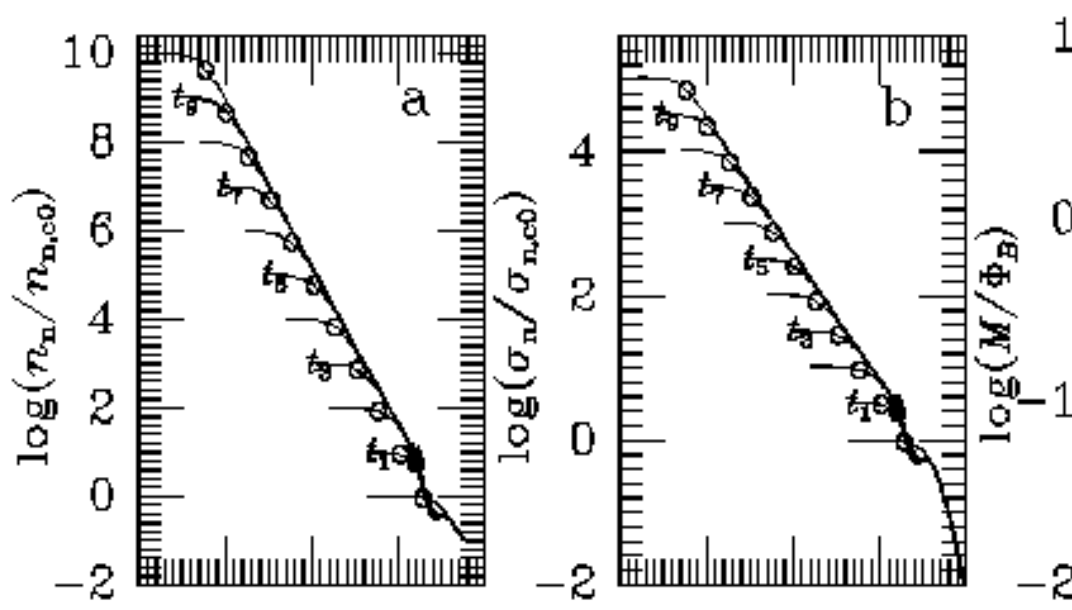

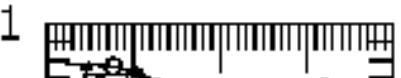

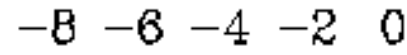

$\begin{array}{lllll}-8 & -6 & -4 & -2 & 0\end{array}$

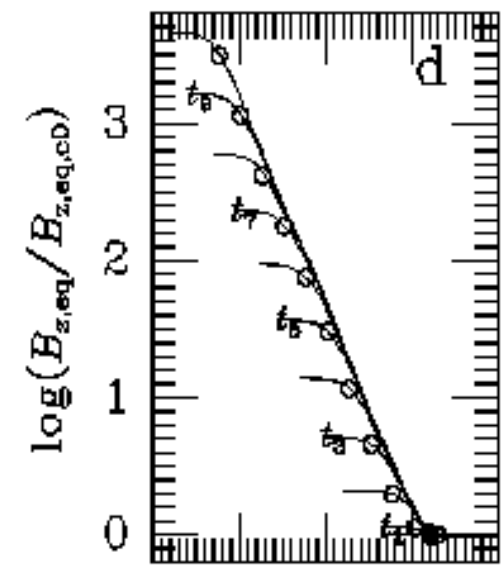

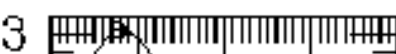

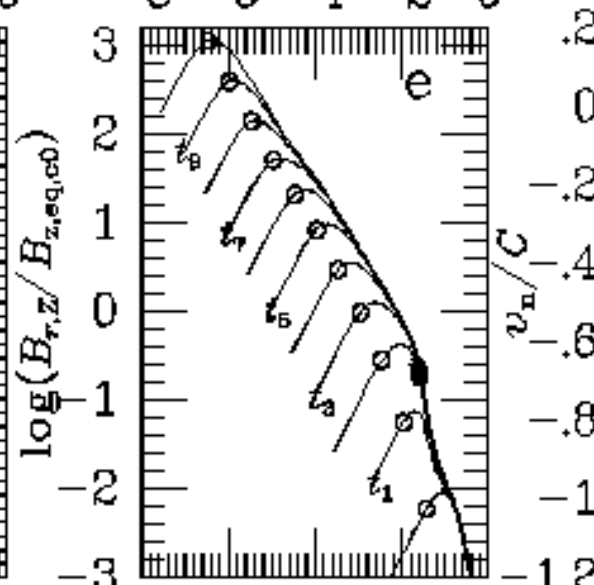

$\begin{array}{lllll}-8 & -6 & -4 & -2 & 0\end{array}$

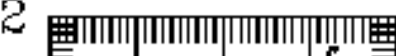

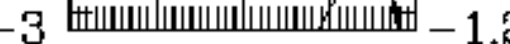

2

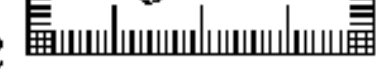

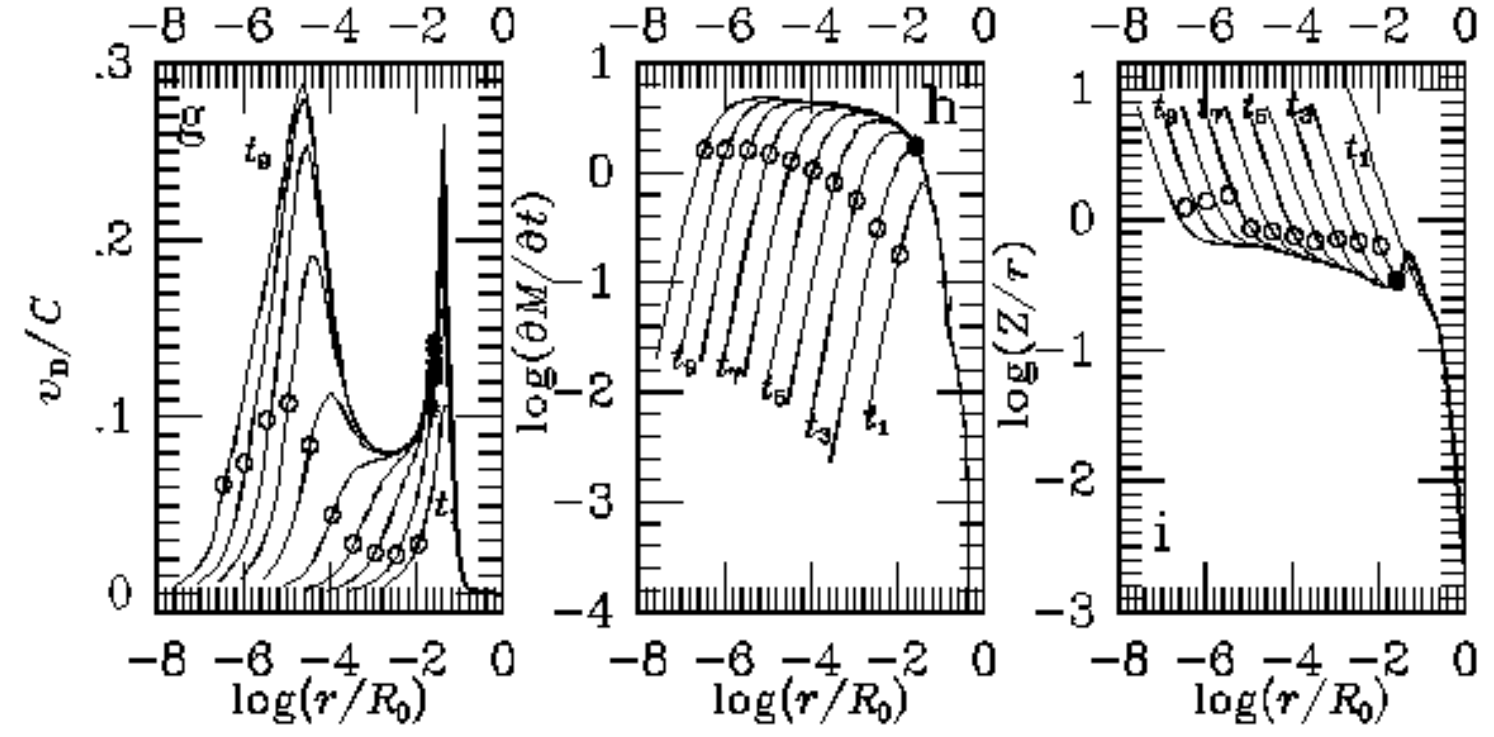

Fig. 1.- 


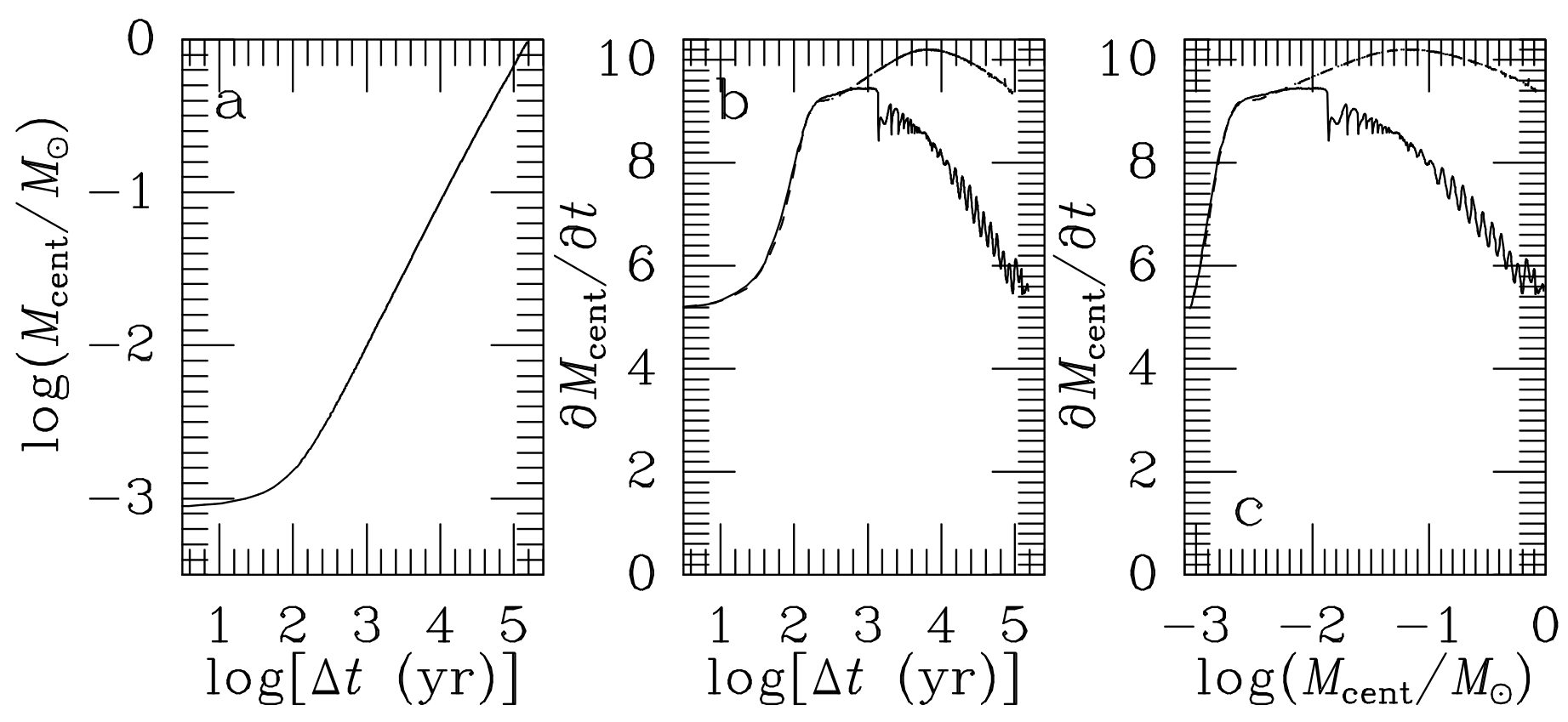

Fig. 2.- 


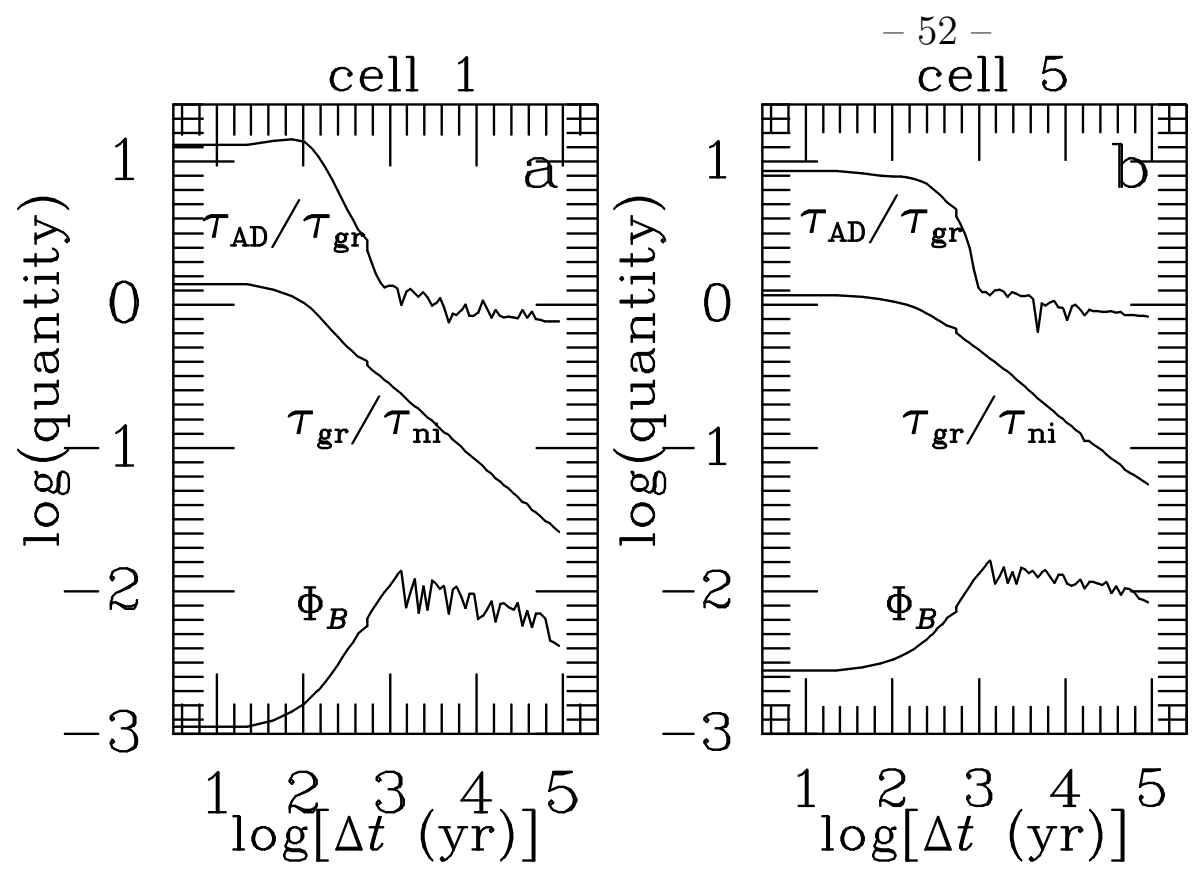

Fig. 3.- 


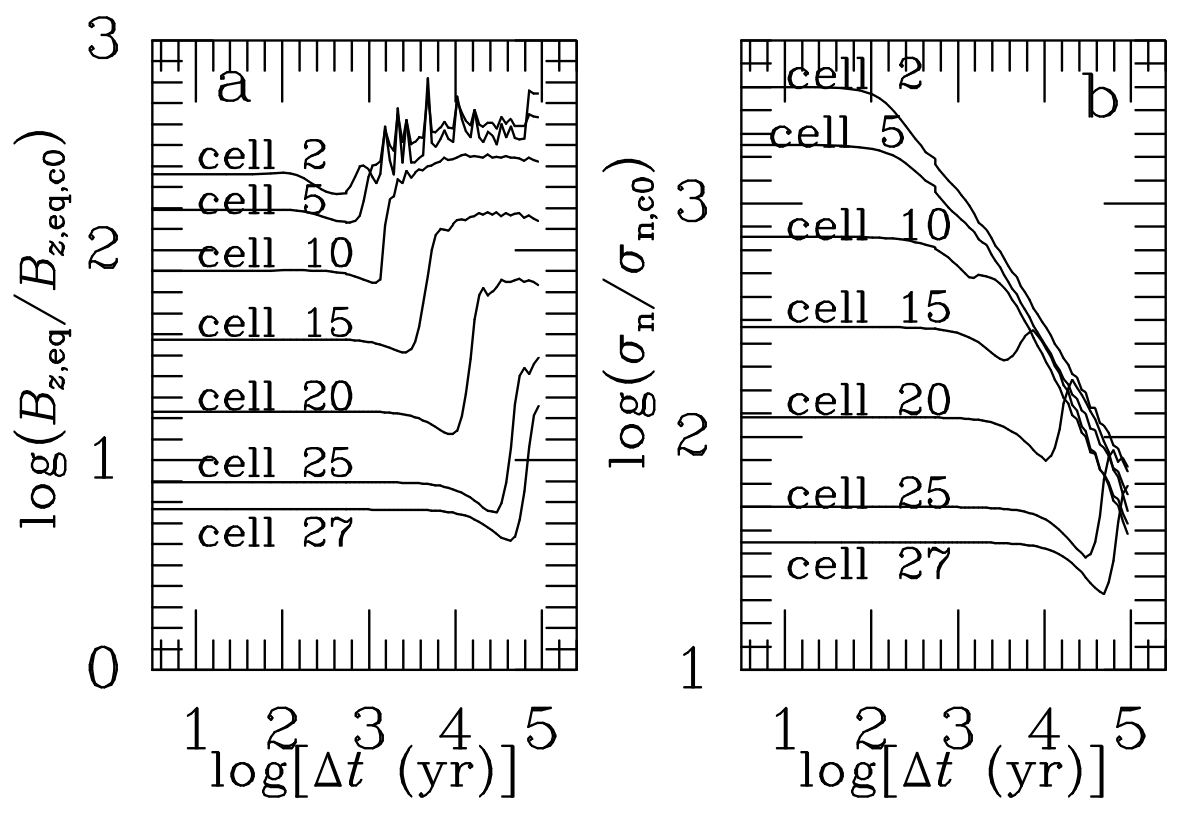

Fig. 4.- 


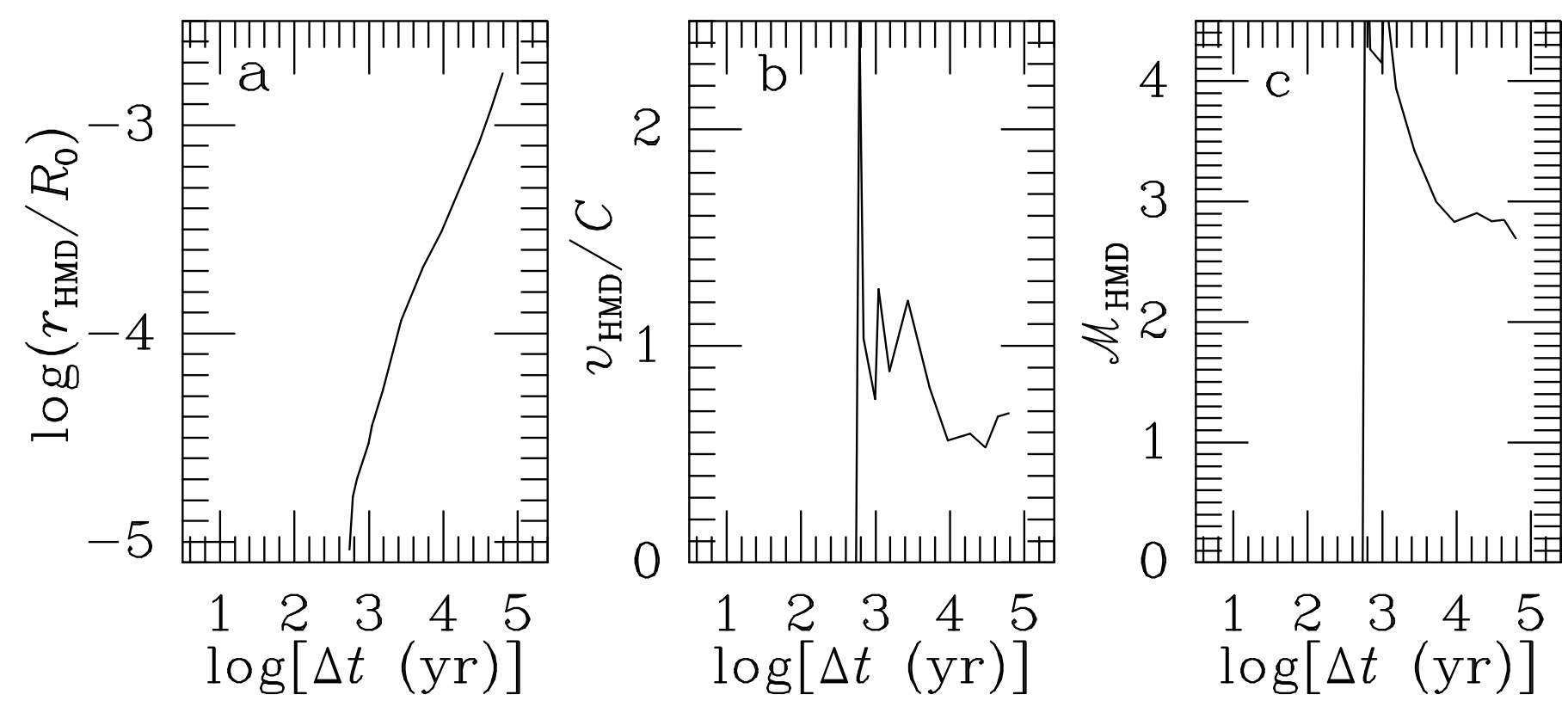

Fig. 5.- 

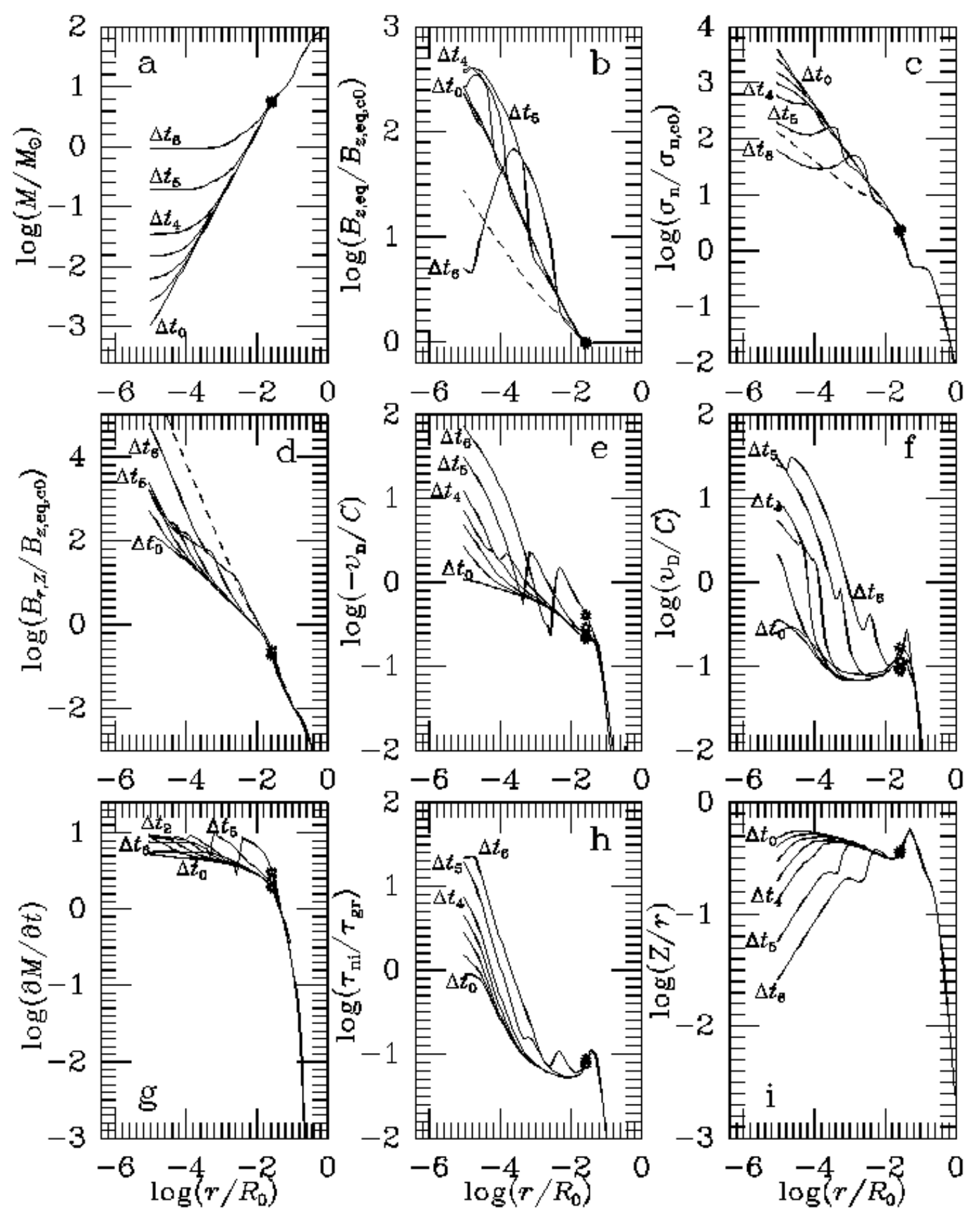

Fig. 6.- 


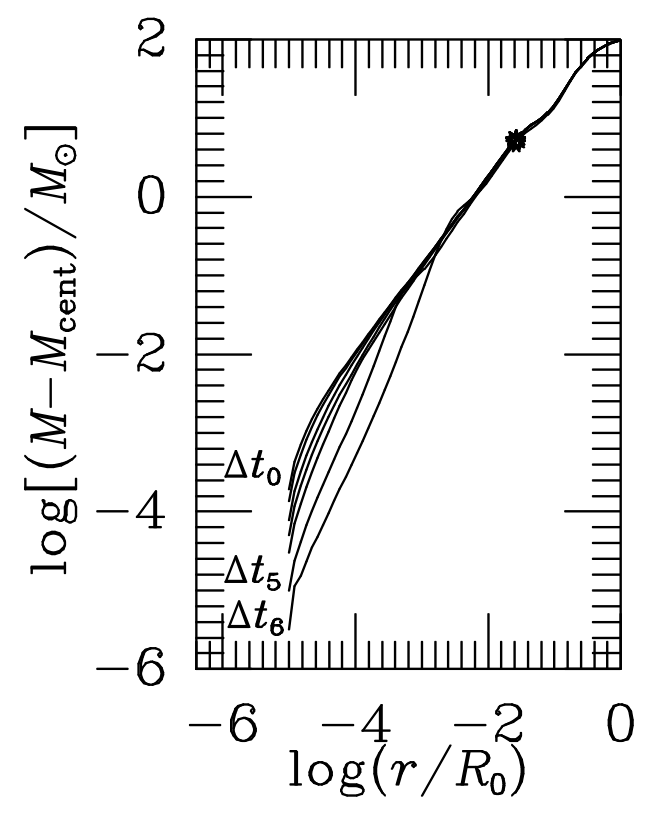

Fig. 7.- 


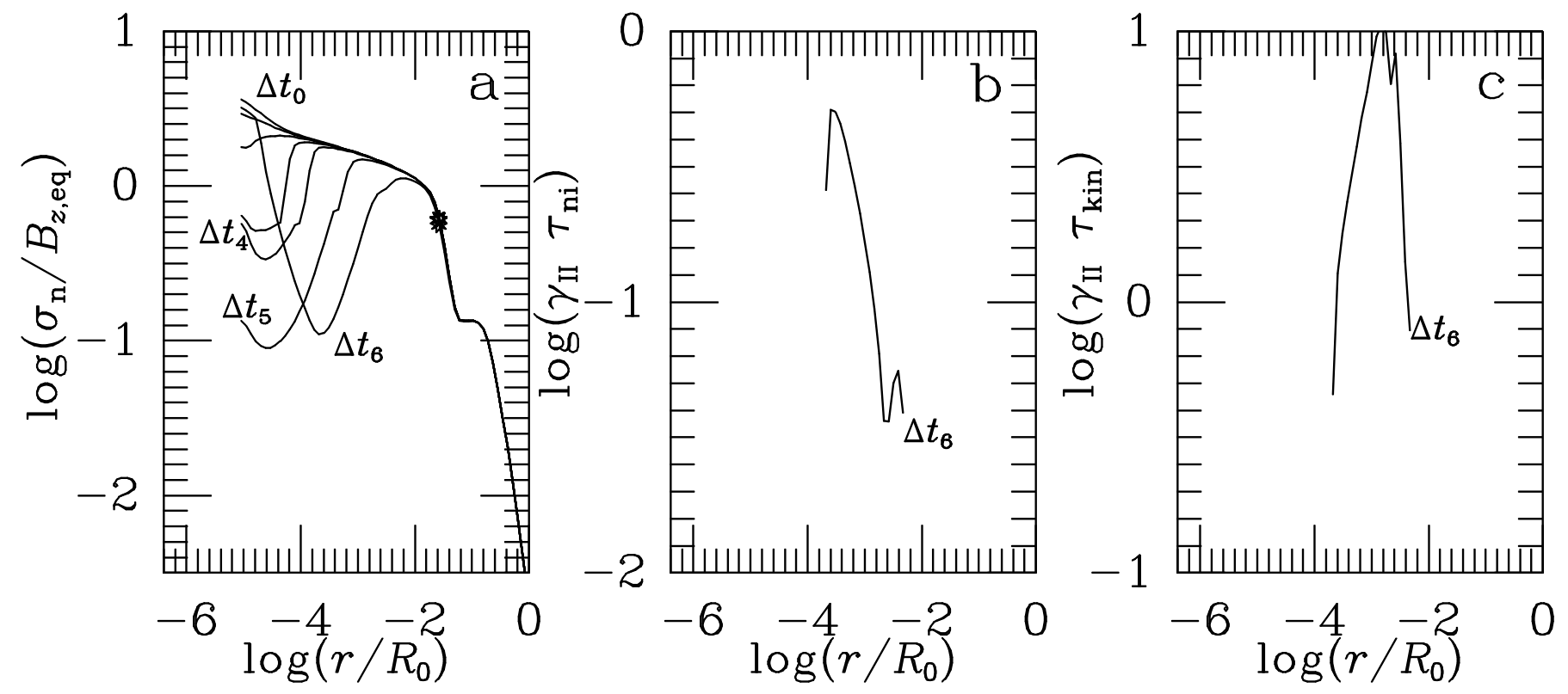

Fig. 8.- 


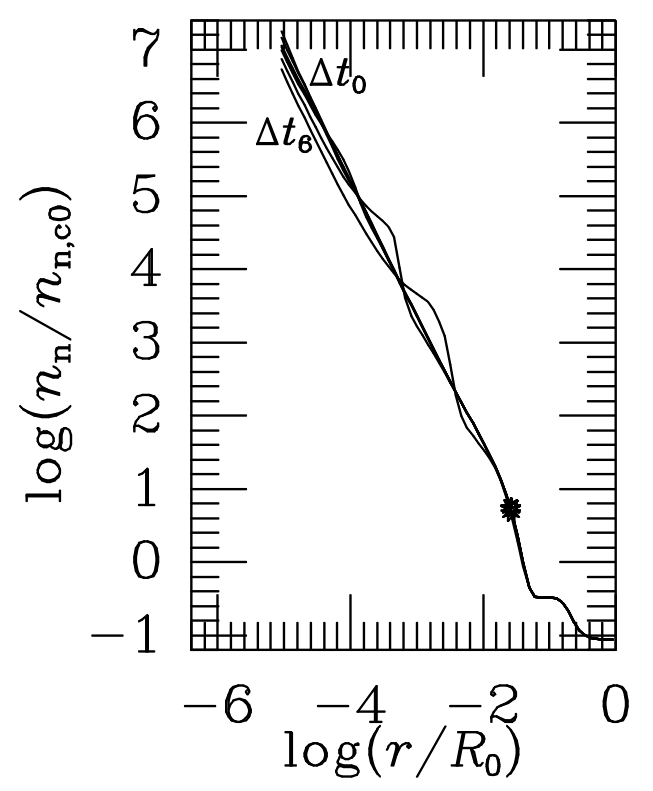

Fig. 9.- 

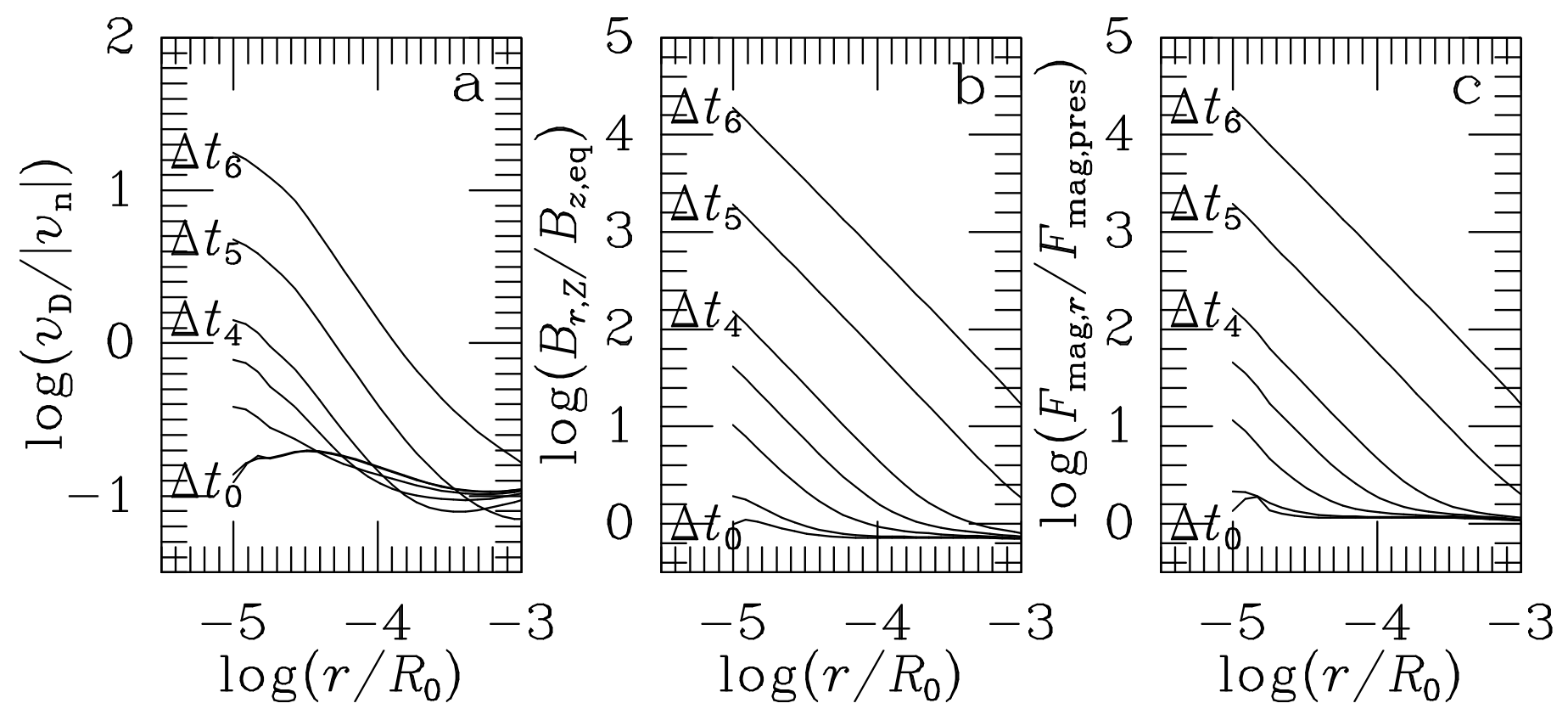

Fig. 10.- 